\title{
Peripheral CB1 receptor blockade acts as a memory enhancer through
}

\section{an adrenergic-dependent mechanism}

Sara Martínez-Torres ${ }^{1,2,3}$, Araceli Bergadà-Martínez ${ }^{1}$, Jorge E. Ortega ${ }^{4,5}$, Lorena GaleraLópez $^{1}$, Arnau Hervera ${ }^{2,3}$, Antonio Ortega-Álvaro ${ }^{1}$, Floortje Remmers ${ }^{6}$, Emma MuñozMoreno $^{7,8}$, Guadalupe Soria ${ }^{7,8}$, José Antonio del Río ${ }^{2,3}$, Beat Lutz ${ }^{6,9}$, Jose Ángel RuízOrtega $^{4}$, J. Javier Meana ${ }^{4,5}$, Rafael Maldonado ${ }^{* 1,10}$, Andrés Ozaita ${ }^{* 1,10}$

${ }^{1}$ Laboratory of Neuropharmacology-NeuroPhar, Department of Experimental and Health Sciences. Universitat Pompeu Fabra, Barcelona, Spain.

${ }^{2}$ Molecular and Cellular Neurobiotechnology, Institute for Bioengineering of Catalonia. Parc Científic de Barcelona, Barcelona, Spain.

${ }^{3}$ Centro de Investigación Biomédica en Red sobre Enfermedades Neurodegenerativas; Department of Cell Biology, Physiology and Immunology, Universitat de Barcelona; Institute of Neuroscience, University of Barcelona, Barcelona, Spain.

${ }^{4}$ Department of Pharmacology, University of the Basque Country UPV/EHU, Leioa, Spain.

${ }^{5}$ Centro de Investigación Biomédica en Red de Salud Mental CIBERSAM, Spain; Biocruces Bizkaia Health Research Institute, Barakaldo, Spain.

${ }^{6}$ Institute of Physiological Chemistry, University Medical Center of the Johannes Gutenberg University of Mainz, Mainz, Germany.

${ }^{7}$ Experimental 7T MRI Unit, Magnetic Resonance Imaging Core Facility (IDIBAPS), Barcelona, Spain.

${ }^{8}$ Laboratory of Surgical Neuroanatomy, Faculty of Medicine and Health Sciences, Institute of Neurosciencies, University of Barcelona, Barcelona, Spain.

${ }^{9}$ Leibniz Institute for Resilience Research (LIR), Mainz, Germany.

${ }^{10}$ IMIM Hospital del Mar Research Institute, Barcelona, Spain.

* Corresponding authors: Andrés Ozaita, Laboratori de Neurofarmacologia, Facultat de Ciències de la Salut i de la Vida, Universitat Pompeu Fabra, Parc de Recerca Biomèdica de Barcelona, C/ Doctor Aiguader 88, 08003 Barcelona, Spain. Phone: +34-93-3160823; Fax:+34-93-3160901; e-mail: andres.ozaita@upf.edu

Rafael Maldonado, Laboratori de Neurofarmacologia, Facultat de Ciències de la Salut i de la Vida, Universitat Pompeu Fabra, Parc de Recerca Biomèdica de Barcelona, C/ Doctor Aiguader 88, 08003 Barcelona, Spain. Phone: +34-93-3160824; Fax: + 34-933160901; e-mail: rafael.maldonado@upf.edu

Keywords: memory persistence; CB1 cannabinoid receptor; peripheral-to-central, adrenergic system, vagus nerve, locus coeruleus 


\begin{abstract}
Peripheral inputs to the brain continuously shape its function and can influence the formation of non-emotional memory, but the underlying mechanisms have not been fully understood. Cannabinoid type-1 receptors (CB1R), widely distributed in the organism, is a well-recognized player in memory performance, and its systemic modulation significantly influences memory function. By assessing non-emotional memory in mice, we have now found a relevant role of peripheral $\mathrm{CB} 1 \mathrm{R}$ in the formation of persistent memory. Indeed, peripherally restricted CB1R antagonism by using AM6545 showed a mnemonic effect that was occluded in adrenalectomized mice, after peripheral adrenergic blockade, or when vagus nerve was chemogenetically inhibited. Genetic CB1R deletion in dopamine $\beta$-hydroxylase-expressing cells enhanced the formation of persistent memory, supporting a role of peripheral CB1R modulating the adrenergic tone. Notably, brain connectivity was affected by peripheral CB1R inhibition, and locus coeruleus activity and extracellular hippocampal norepinephrine, were increased. In agreement, intra-hippocampal $\beta$-adrenergic blockade prevented AM6545 mnemonic effects. Together, we disclose a novel peripheral mechanism relevant for the modulation of the formation of persistent non-emotional memory.
\end{abstract}




\section{Introduction}

Most everyday experienced events create non-emotional memories with different lifespans. They are generally rather short-lived and may fade away or may persist for longer periods, with the intervention of the hippocampus and further recall, even if all short- and long-lived memories were created from similar sensorial stimuli (Morris, 2006). Therefore, the persistence of stimuli-driven memories involves a discrimination/selection of worth-memorizing stimuli, which may occur around the time of encoding (Kandel et al., 2014; Yonelinas et al., 2019). Such memory persistence is enhanced in emotionally arousing experiences, where encoding is combined with the natural stress-coping response, which leads to a significant increase in memory persistence for the sensory information recorded at that time (De Quervain et al., 2016). In contrast, the mechanisms involved in memory persistence for non-emotional memories are not well understood, although they are also relevant for everyday life events.

The endocannabinoid system (ECS), highly expressed in the central nervous system (CNS) and peripheral tissues (Kano et al., 2009; Maccarrone et al., 2015), plays a key role in learning and memory (Kano et al., 2009). The cannabinoid type-1 receptor (CB1R) is strongly expressed in the brain (Pacher et al., 2006), predominantly localized at presynaptic sites of different neuronal cell types, where it suppresses neurotransmitter release depending on local synaptic activity (Castillo et al., 2012). Exogenous compounds with agonist properties for CB1R contribute to memory impairment (Niyuhire et al., 2007; Puighermanal et al., 2012) in mice in non-emotional memory medels, such as the novel object-recognition test (NORT), while pharmacological or genetic CB1R blockade increases memory persistence in these models (Maccarrone et al., 2002; Reibaud et al., 1999). Although the mechanisms involved are largely unknown, such regulation of 
memory by CB1R blockade was previously assumed to occur solely through centrally located receptors (Zanettini et al., 2011).

We set to challenge this dogma by studing the mnemonic effect of a peripherally restricted CB1R antagonist, AM6545 (Tam et al., 2010). Peripheral CB1R had been previously found relevant for the amnesic effects associated to stress at the time of memory consolidation (Busquets-Garcia et al., 2016), but their contribution to memory enhancement had not been previously assessed.

We found that peripheral blockade of CB1R on adrenergic cells enhanced non-emotional memory persistence through an adrenergic mechanism involving the vagus nerve. Under these conditions, brain connectivity was increased and hippocampal norepinephrine release was boosted as a plausible mechanism underlying this enhancement of memory persistence. 


\section{Results}

$C B 1 R$ inhibition enhances memory persistence in the novel object-recognition memory test

Novel object-recognition memory is a labile non-emotional type of memory usually evaluated $3 \mathrm{~h}$ or $24 \mathrm{~h}$ after the familiarization session, when mice readily discriminate novel and familiar objects (Fig. 1a). Notably, discrimination values significantly decrease when novel object-recognition memory is assessed $48 \mathrm{~h}$ after the familiarization phase (one-way ANOVA, interaction: $\mathrm{F}(2,19)=8.55, \mathrm{p}=0.002$; post hoc Tukey, $3 \mathrm{~h}$ vs $48 \mathrm{~h} \mathrm{p}$ $=0.003 ; 24 \mathrm{~h}$ vs $48 \mathrm{~h} \mathrm{p}=0.007)($ Fig. $1 \mathrm{a})$. We used this non-emotional memory paradigm assayed $48 \mathrm{~h}$ after the familiarization phase to evaluate the role of CB1R inhibition in memory persistence. We found that mice with acute post-familiarization treatment with a low dose of the systemic CB1R selective antagonist rimonabant $(1 \mathrm{mg} / \mathrm{kg}$, i.p. $)$ showed higher memory persistence than vehicle-treated mice (Student's t-test: $p=0.02$ ) (Fig. 1c). In addition, heterozygous mice for the Cnrl gene (CB1HZ) also showed enhanced memory persistence (Student's t-test: $\mathrm{p}=0.004)$ compared to their wild-type littermates (Fig. 1d), indicating that such a modulation in memory persistence is CB1R dependent. Post-familiarization administration of the peripherally-restricted CB1R antagonist AM6545 (1 mg/kg, i.p.) also enhanced novel object-recognition memory at $48 \mathrm{~h}$ (Student's t-test: $\mathrm{p}=0.002$ ) (Fig. 1e) pointing to the involvement of a peripheral mechanism. No differences in total exploration time were detected between genotypes or pharmacological treatments in any of the experimental groups above (Supplementary Fig. 1) discarding a possible bias due to differences between groups in exploratory behavior. Furthermore, AM6545 treatment did not affect locomotor activity analyzed for $120 \mathrm{~min}$ post-administration (Supplementary Fig. 2) excluding a major unspecific effect of the treatment on behavioral responses. 
Enhanced memory persistence of peripheral CB1R inhibition involves a peripheral adrenergic mechanism

We hypothesized that a peripherally located tissue, such as the adrenal glands which express CB1R (Hillard, 2015), could be a relevant player modulating memory consolidation (McIntyre et al., 2012). Therefore, we evaluated the effect of postfamiliarization AM6545 administration in bilaterally adrenalectomized mice. Memory persistence enhancement by AM6545 was significantly reduced in mice without adrenal glands (two-way ANOVA, interaction: $\mathrm{F}(1,22)=4.89, \mathrm{p}=0.037$; post hoc Tukey, naiveVEH vs naive-AM6545, $\mathrm{p}=0.022$; naive-AM6545 vs ADX-AM6545, $\mathrm{p}=0.026)($ Fig. 2a), supporting the role of CB1R blockade in this paticular peripheral tissue. Adrenal glands release glucocorticoids and catecholamines into the blood, both relevant for memory (McIntyre et al., 2012). In addition, both rimonabant (Wade et al., 2006) and high doses of AM6545 have been observed to increase circulating corticosteroids (Roberts \& Hillard, 2020). To elucidate out which hormones are responsible for the mnemonic effects produced by peripheral CB1R blockade, mice were pre-treated after the familiarization phase with the glucocorticoid receptor antagonist mifepristone (50 $\mathrm{mg} / \mathrm{kg}$ i.p.) or the peripherally-restricted $\beta$-adrenergic receptor antagonist sotalol (10 $\mathrm{mg} / \mathrm{kg}$ i.p.) $20 \mathrm{~min}$ before AM6545 injection (Fig. 2b). Mifepristone pre-treatment did not prevent enhancement of memory persistence by AM6545 (two-way ANOVA, interaction: $\mathrm{F}(1,21)=0.038, \mathrm{p}=0.845$; mifepristone/vehicle effect: $\mathrm{F}(1,21)=0.707, \mathrm{p}$ $=0.409$; AM6545/vehicle effect: $F(1,21)=25.11, p<0.001)$ (Fig. 2b). In contrast, mice pre-treated with sotalol did not show the memory improvement observed in AM6545treated mice (two-way ANOVA, interaction: $\mathrm{F}(1,31)=7.58, \mathrm{p}=0.009$; post hoc Tukey, Saline-VEH vs Saline-AM6545 p =0.01; Saline-AM6545 vs Sotalol-AM6545 p =0.001) (Fig. 2c). 
In the light of these data, we assessed whether inhibition of CB1R exclusively in dopamine $\beta$-hydroxylase cells $(\mathrm{DBH}+$ cells $)$, the main cells responsible for circulating levels of epinephrine/norepinephrine, could mimic the mnemonic effect of systemic and peripheral CB1R antagonists. We used a combination of genetic and pharmacological approaches to first show that conditional knock-out mice lacking the CB1R in DBH+ cells (DBH-CB1KO mice) displayed enhanced novel object-recognition memory persistence compared to wild-type controls (Student's t-test: $p=0.04$ ) (Fig. 2d). In addition, enhanced memory persistence in DBH-CB1KO mice was abolished by sotalol administration (two-way ANOVA, interaction: $\mathrm{F}(1,22)=10.47, \mathrm{p}=0.003$; post hoc Tukey, Saline-WT vs Saline-DBH-CB1KO p = 0.01; Saline- DBH-CB1KO vs SotalolDBH-CB1KO $\mathrm{p}=0.04$ (Fig. 2e), pointing to a relevant role of CB1R-modulated peripheral adrenergic/noradrenergic tone in memory persistence. Sotalol pre-treatment similarly prevented the cognitive improvement elicited by systemically-acting rimonabant supporting the relative prominence of peripheral CB1R blockade in this effect of rimonabant (two-way ANOVA, interaction: $\mathrm{F}(1,37)=9.408, \mathrm{p}=0.004$; post hoc Tukey, Saline-VEH vs Saline-rimonabant $\mathrm{p}=0.01$; Saline-rimonabant vs Sotalolrimonabant $\mathrm{p}=0.001)$ (Fig. 2f). No differences in total exploration time were detected in the memory test between genotypes or pharmacological treatments in any of the experimental groups above (Supplementary Fig. 3).

Chemogenetic vagus nerve inhibition prevents memory improvement produced by peripheral CB1R blockade

Systemic epinephrine administration does not cross the blood brain barrier and enhances hippocampal dependent memory in rodents by the activation of peripheral $\beta$-adrenergic receptors (Dornelles et al., 2007). Such mnemonic effects have been hypothesized to 
occur either by the activation of $\beta$-adrenergic receptors in the liver and the subsequent increase of blood glucose levels, or by the activation of $\beta$-adrenergic receptors on the afferent fibers of the vagus nerve (Gold \& Korol, 2012).

To further elucidate whether either of these two mechanisms could be involved in the mnemonic effects produced by acute AM6545 administration, we first measured blood glucose levels after AM6545 administration in mice. No differences in blood glucose levels were observed in mice treated with AM6545 in comparison to vehicle-treated group (Fig. 3a).

We used a chemogenetic approach to selectively reduce the neural activity of vagus nerve and assess the involvement of vagal fibers as a major link between peripheral AM6545 effects and memory performance We first confirmed that AM6545 effect on memory persistence was maintained in control mice receiving bilateral vagus nerve injection of AAV5-mCherry and subsequently treated with clozapine $\mathrm{N}$-oxide (CNO, $3 \mathrm{mg} / \mathrm{kg}$, i.p.) (Fig. 3b), indicating that surgery, vagus nerve infection with viral vectors, and CNO administration were compatible with the assessment of memory persistence. However, chemogenetic inhibition in animals with bilateral vagus nerve injection of AAV5-hM4Di prevented AM6545-memory enhancement (Fig. 3c) revealing a role of vagus nerve afferents in the mnemonic effect of AM6545. As expected, no differences were observed neither when memory was evaluated $24 \mathrm{~h}$ after familiarization in AAV5-hM4Di mice nor in exploratory behavior during the memory test (Supplementary Fig. 4). Thus, AM6545 administration improves memory consolidation through an adrenergic mechanism involving the vagus nerve. 
We first analyzed the pattern of c-Fos expression, as a marker of neuronal activity, focusing on brain regions relevant for memory performance. Samples were obtained 90 min after receiving AM6545 or vehicle once mice has finished the familiarization phase in the NORT. Brain areas analysed included CA1 and CA3 hippocampal regions, dentate gyrus, prelimbic and infralimbic prefrontal cortex, locus coeruleus and basal, lateral and central regions of the amygdala. No significant differences in c-Fos expression were observed between AM6545- and vehicle-treated mice in the areas analyzed (Supplementary Fig.5). However, network analysis using the Pearson correlation coefficient for each pair of regions revealed that AM6545 treatment produced a significant alteration in the functional connectivity of these brain areas compared to the vehicle condition. (Fig. 4a-b and Supplementary Fig.6). We calculated Z-scores starting from the Pearson $r$ correlation values to compare positive and negative connectivity between groups. AM6545 treatment did not significantly modified neither positive nor negative correlations (Fig. 4c-d).

We further explored the central effects after peripheral CB1R blockade by performing resting state functional magnetic resonance imaging (rsfMRI). After 1 hour of AM6545 administration, global connectivity metrics showed a significant enhancement of AM6545 treatment in whole brain local efficiency (Fig. 5a) (uncorrected p<0.05) indicating an enhanced ability of parallel transmission of information. Regional connectivity analysis showed significant increases of nodal efficiency values in AM6545treated mice compared to vehicle-treated mice in left frontal cortex (Kruskal-Wallis: $\mathrm{p}<0.01$ ), right hippocampus (Kruskal-Wallis: $\mathrm{p}=0.023$ ) and right globus pallidus (Kruskal-Wallis: $p=0.023$ ) (Fig. 5 b-d), although these differences did not reach significance when corrected for multiple comparisons (FDR), indicating a subtle effect of AM6545 enhancing brain connectivity. Seed-based analysis of rsfMRI with seed in the 
posterior brainstem, which contains the nucleus tractus solitarius (NTS), a region receiving vagal afferents, exhibited a different pattern of connectivity in AM6545-treated animals compared to vehicle-treated animals. Significant differences of this connectivity pattern were observed in areas corresponding to hippocampus, frontal cortex, cingulate cortex, brainstem nuclei such as pontine reticular nucleus, several preolivary and spinal trigeminal nuclei and cerebellar nuclei (Fig. 5e). In these regions, AM6545-treated animals showed strong negative correlation values with the seed BOLD signal, especially in hippocampus, that are not observed in vehicle treated animals (Supplementary Fig.7).

Increased hippocampal norepinephrine mobilization by peripheral CBIR blockade induces memory improvement

Next, we measured the firing rate at locus coeruleus neurons after CB1R peripheral modulation with AM6545. Systemic AM6545 produced a non-significant enhancement of basal firing rates compared to vehicle (Fig. 6a). To assess the functional significance of the basal firing rate, we performed extracellular microdialysis analysis in the hippocampus after systemic AM6545 treatment. Analysis of norepinephrine (NE), dopamine (DA) and serotonin (5-HT) extracellular levels after AM6545 administration revealed a specific transient increase in NE in comparison to the vehicle-treated mice (two-way repeated measures ANOVA, interaction: $F(1,14)=2.19 \mathrm{p}=0.009)$ (Fig. 6b and Supplementary Fig.8). We then tested whether $\beta$-adrenergic receptors in the hippocampal region were involved in the increased memory persistence mediated by AM6545. We found that local intra-hippocampal microinjection of propranolol at a dose that did not affect memory performance on its own $(1 \mu \mathrm{g}$ per $0.5 \mu \mathrm{L}$ per side, see Supplementary Fig. 9) blocked the mnemonic effects produced by systemic AM6545 administration (two-way ANOVA, interaction: $\mathrm{F}(1,119)=5.03, \mathrm{p}=0.03$; post hoc 
Tukey, Saline-VEH vs Saline-AM6545 p =0.004; Saline-AM6545 vs PropranololAM6545 $\mathrm{p}=0.01$ ) (Fig. 6c), without affecting the exploratory behavior on the memory test (Fig. 6d). These data indicate the functional relevance of noradrenergic hippocampal activation in the effect of peripheral CB1R blockade on memory persistence. 


\section{Discussion}

Our study identifies a relevant role of the peripheral ECS in modulating non-emotional memory persistence through the mobilization of central and peripheral adrenergic/noradrenergic mechanisms. We chose to study novel object-recognition memory, a hippocampal-dependent test (Cohen \& Stackman, 2015) since this is a model of non-emotional memory and memory persistence might be modulated in this test by post-training manipulation. In this regard, we have previously observed deficits in novel object-recognition memory by CB1R agonists (Puighermanal et al., 2009), endocannabinoid build-up (Busquets-Garcia et al., 2011) and stress (Busquets-Garcia et al., 2016), all interventions after the familiarization phase. In the present study, we analyzed novel object-recognition memory recall $48 \mathrm{~h}$ after the familiarization phase since at that time mice usually show reduced signs of novel object discrimination compared to shorter intervals. Using this $48 \mathrm{~h}$ interval, we found that overall CB1R blockade, through pharmacological or genetic approaches, significantly increased discrimination indexes. In agreement with this, a number of previous studies had also demonstrated that both pharmacological and genetic CB1R inactivation enhanced memory in different hippocampal-dependent tasks (Jacob et al., 2012; Lichtman, 2000; Maccarrone et al., 2002; Reibaud et al., 1999; Takahashi et al., 2005; Wolff \& Leander, 2003). We found that the peripherally restricted CB1R neutral antagonist AM6545 administered after the familiarization phase significantly enhanced object-recognition memory persistence in mice. This drug does not show significant blood-brain barrier permeability at doses even ten times higher than the one used in the present study (Tam et al., 2010), suggesting that the AM6545 effects derive in our study from a peripheral mechanism. Notably, adrenalectomized mice treated with AM6545 did not present an enhancement in memory persistence, pointing to a relevant role of adrenal glands in this 
response. Epinephrine/norepinephrine and corticosteroids secreted by the adrenal glands have a significant impact in memory consolidation (McIntyre et al., 2012; Roozendaal \& McGaugh, 2011; Yang et al., 2013). We then gathered evidence that pointed to the mobilization of the adrenergic transmission by peripheral CB1R blockade, but not to the mobilization of corticosteroids, given that AM6545-induced memory persistence enhancement was prevented by the peripherally restricted $\beta$-adrenergic receptor antagonist sotalol, but not by the corticosteroid receptor antagonist mifepristone. Such differential modulation by adrenal hormones of AM6545 effect is indicative of a rather specific mechanism of peripheral CB1R blockade for potentiating the consolidation of non-emotional memory. Indeed, sotalol has been previously found ineffective in modulating fear conditioning (Lee et al., 2001) and mifepristone was found to reduce contextual memory in a fear conditioning paradigm (Zhou et al., 2010), whereas it did not modify NORT results in our experimental conditions. Notably, mice lacking CB1R in $\mathrm{DBH}+$ cells (Busquets-Garcia et al., 2016) (DBH-CB1KO) reproduced a similar phenotype on enhanced memory persistence as constitutive $\mathrm{CB} 1 \mathrm{HZ}$ mice. Our present finding that sotalol prevents such a mnemonic phenotype in DBH-CB1 KO mice and after systemic rimonabant treatment, points to a relevant effect of the peripheral adrenergic CB1R in the systemic effect of CB1R inhibition, and a hitherto undisclosed role of peripheral CB1R signaling in memory persistence.

The study of c-Fos expression after AM6545 administration allowed to confirm no major differences in neuronal stimulation due to the treatment, but significant alterations in functional connectivity between areas analyzed. Although c-Fos may not reveal the real activity of brain regions given its limited expression and the time constraints of the analysis (McReynolds et al., 2018), this approach has been successfully performed to acquire a glimpse of brain functional connectivity between discrete areas (Silva et al., 
2019; Tanimizu et al., 2017). The subtle alterations in brain connectivity revealed by our biased c-Fos inter-regional correlation analysis were further corroborated by rsfMRI using an unbiased approach to study connectivity. The brain's intrinsic functional organization associated to the resting state has been proposed to determine the ability to create flexible and suitable behavioral outcomes to cognitive demands (Sala-Llonch et al., 2012). Indeed, enhancements in nodal efficiency, a representation for the ability of information propagation in the subnetwork of regions connected to a node, were observed specifically in the frontal cortex, hippocampus and globus pallidus, a result in agreement with the engagement of these regions in long-term memory consolidation (Kitamura et al., 2017; Tanimizu et al., 2017) promoted by AM6545 treatment.

A growing body of evidence has demonstrated the efficacy of systemic epinephrine administration, which does not cross the blood-brain barrier, to enhance hippocampaldependent memory in rodents (Dornelles et al., 2007; Talley et al., 2000) by enhancing central noradrenergic signaling. Our data reveal that AM6545 administration did modestly modify brain activity to produce a reduction in brain network connectivity and a partial increase in basal LC firing and norepinephrine extracellular levels in the hippocampus, suggesting increased activity of LC projections to this brain region. These finding are compatible with the activation of the vagus nerve by increasing peripheral adrenaline/noradrenaline, since it was demonstrated that epinephrine administration results in increased vagal nerve firing that was sensitive to sotalol administration (Miyashita \& Williams, 2006). Vagus nerve stimulation has been found to enhance memory retention in mouse models (Vázquez-Oliver et al., 2020) as well as in humans (Ghacibeh et al., 2006). LC activity, partially determined by the vagal nerve afferents, is well stablished to be physiologically engaged on novelty and to prime the persistence of hippocampal-based long-term memories (Hansen, 2017; Sara, 2009). Moreover, 
norepinephrine in the hippocampus facilitates the storage of new memories through the regulation of neural excitability and synaptic plasticity (Hagena et al., 2016). Although norepinephrine can bind to both $\alpha$ - and $\beta$-adrenergic receptors, synaptic information and plasticity in the hippocampus is proposed to depend largely on the activation of $\beta$ adrenergic receptors (Kemp \& Manahan-Vaughan, 2008). Indeed, LC stimulation modulates hippocampal synaptic strength (Hansen \& Manahan-Vaughan, 2015; Kemp \& Manahan-Vaughan, 2012, 2008), spike coupling of CA1 pyramidal neurons (Bacon et al., 2020) and improves memory through a $\beta$-adrenergic-dependent mechanism (Hagena et al., 2016; Hansen \& Manahan-Vaughan, 2015). In agreement, we observed that the intrahippocampal blockade of $\beta$-adrenergic receptors with propranolol prevented the increase of novel object-recognition memory persistence produced by AM6545.

Altogether, our study identifies peripheral CB1R as a relevant target to enhance nonemotional memory persistence through a central and peripheral adrenergic mechanism that engages the vagus nerve. This novel peripheral mechanism involving CB1R could underlie previously described effects of systemic CB1R antagonists previously assumed to be associated to direct interaction with central targets. 


\section{Materials and Methods}

\section{Animals}

Young adult (10-12 weeks old) male Swiss albino (CD-1) mice (Charles River, France) were used for pharmacological approaches in behavioural, microdialysis, electrophysiological and biochemical experiments. For genetic approaches to reduce CB1R expression, heterozygous mice for the Cnrl gene (CB1HZ) and their wild-type littermates in C57BL/6J genetic background were used (Zimmer et al., 1999). Conditional KO mice for the Cnrl gene lacking CB1R exclusively in DBH-expressing cells were generated as previously detailed (Busquets-Garcia et al., 2016) in C57BL/6J genetic background.

Mice were housed in Plexiglas cages (2-4 mice per cage) and maintained in temperature $\left(21 \pm 1^{\circ} \mathrm{C}\right)$ and humidity $(55 \pm 10 \%)$ controlled environment. Food and water were available ad libitum. All the experiments were performed during the light phase of a $12 \mathrm{~h}$ cycle (light on at $8 \mathrm{am}$; light off at $8 \mathrm{pm}$ ). Mice were habituated to the experimental room and handled for 1 week before starting the experiments. All behavioural experiments were conducted by an observer blind to experimental conditions.

All animal procedures were conducted following "Animals in Research: Reporting Experiments" (ARRIVE) guidelines and standard ethical guidelines (Kilkenny et al., 2010) (European Directive 2010/63/EU). Animal procedures were approved by the local ethical committee (Comitè Ètic d'Experimentació Animal-Parc de Recerca Biomèdica de Barcelona, CEEA-PRBB).

\section{Drugs and treatments}

Rimonabant (Axon Medchem) and mifepristone (Sigma-Aldrich) were dissolved in 5\% ethanol, 5\% cremophor-EL and 90\% saline (0.9\% NaCl). AM6545 (Tocris-Bio-Techne) 
was dissolved in $0.26 \%$ DMSO, 4.74\% ethanol, 5\% cremophor-EL and 90\% saline. Sotalol (Sigma-Aldrich) was dissolved in saline. Propranolol (Sigma-Aldrich) was dissolved in saline. For the activation of the inhibitory designer receptors exclusively activated by designer drugs (hM4Di-DREADD), clozapine N-oxide (CNO) (Enzo Life Sciences, NY) was dissolved in sterile saline. All intraperitoneally (i.p.)-injected drugs were administered in a volume of $10 \mathrm{~mL} / \mathrm{kg}$ of body weight at the doses and time points indicated.

\section{Viral vectors}

We used the following vectors: AAV-hM4Di-DREADD (AAV5- hSyn-hM4D(Gi)mCherry) and AAV-control-DREADD (AAV5-hSyn-mCherry) from Addgene.

To detect the viral expression in all the experiments we visualized through epifluorescence microscopy the reporter mCherry in the nodose ganglion.

\section{Bilateral adrenalectomy}

Mice were anesthetized by isoflurane inhalation, $5 \% \mathrm{v} / \mathrm{v}$ induction and 3\% v/v for maintenance, with oxygen $(0.8 \mathrm{~L} / \mathrm{min})$. A small incision of $1 \mathrm{~cm}$ was made in the left and right flanks, and the adrenal glands were identified and removed from the surrounding tissue. Wounds were closed in two layers using 4/0 silk sutures (Alcon). All animals were given access to saline after surgery to ensure adequate salt balance. The experiments were resumed following a recovery period of $10 \mathrm{~d}$.

\section{Bilateral intra-hippocampal cannula implantation}

Bilateral intra-hippocampal cannula implantation was performed as previously described (Busquets-Garcia et al., 2018). Briefly, mice were deeply anaesthetized with a mixture of 
ketamine hydrochloride (Imalgène; Merial Laboratorios S.A.) and medetomidine hydrochloride (Domtor; Esteve, Spain) dissolved in sterile $0.9 \%$ physiological saline and administered intraperitoneally (i.p., $75 \mathrm{mg} / \mathrm{kg}$, and $1 \mathrm{mg} / \mathrm{kg}$ of body weight respectively). Mice were placed in a stereotaxic frame (David Kopf, Tujunga, CA) and an incision was made over the skull and burr holes were drilled with the size of the guide cannula. A bilateral 26-gauge guide cannula (Plastics One, Roanoke, VA) was implanted into the dorsal hippocampus as a guide for a bilateral injection cannula (33-gauge internal cannula, Plastics One). The bilateral guide cannula was fixed using dental cement (Dentalon plus, Heraeus Kulzer GmbH, Hanau, Germany) and two stainless-steel screws. The placement was set at $1 \mathrm{~mm}$ above the target injection site and the guide cannula was sealed with a dummy of stainless-steel wire with $0.5 \mathrm{~mm}$ of projection to prevent obstruction. The target injection site coordinates were as follows: anteroposterior, $-1.80 \mathrm{~mm}$; mediolateral, $\pm 1.00 \mathrm{~mm}$; dorsoventral, $2.00 \mathrm{~mm}$ relative to bregma (Paxinos and Franklin, 2001).

After surgery, anesthesia was reversed by s.c. atipamezole administration (Antisedan, Ecuphar, Spain). Moreover, mice received i.p. administration of gentamicine and s.c. administration of meloxicam.

Animals were kept on a $37^{\circ} \mathrm{C}$ heating pad during the surgery, and until recovery from anesthesia. The behavioral experiments started 1 week after surgery.

\section{Intra-hippocampal drug administration}

After the familiarization phase of NORT, mice received a bilateral intra-hippocampal injection of $0.50 \mu \mathrm{L}$ of propranolol $(0.5,1$ or $2 \mu \mathrm{g})$ or saline at a constant rate of $0.25 \mu \mathrm{L} / \mathrm{min}$ by using a microinfusion pump during $2 \mathrm{~min}$. The injection cannula projected $1.00 \mathrm{~mm}$ below the ventral tip of the implanted guide cannula. The displacement 
of an air bubble inside the length of the polyethylene tubing that connected the Hamilton syringe to the injection needle was used to monitor the microinjections. After infusion, the injection cannula was left for an additional period of 2 min to allow the fluid to diffuse and to prevent reflux before withdrawal.

\section{Verification of cannula position}

After behavioural evaluation, mice were killed by cervical dislocation and the brains removed and frozen at $-80^{\circ} \mathrm{C}$. Coronal slices were obtained on a cryostat, mounted on slides and stained with cresyl violet. The injection sites were verified using a light microscope in blinding conditions. All mice with cannula located outside the hippocampus were excluded from the experiment.

Surgery and virus vector microinjection in the vagus nerve

Mice were anesthetized with a mixture of ketamine hydrochloride (Imalgène; Merial Laboratorios S.A.) and medetomidine hydrochloride (Domtor; Esteve, Spain) dissolved in sterile physiological saline and administered intraperitoneally (i.p., $75 \mathrm{mg} / \mathrm{kg}$, and 1 $\mathrm{mg} / \mathrm{kg}$ of body weight respectively) and placed in a supine position. A small incision from the larynx to approximately the beginning of the sternum was made in the left and right flanks. Submaxillary glands were esposed by blunt dissection and the midline was opened to expose the trachea. Vagus nerve and the carotid artery were identified within the set of neurovascular fibers parallel to the trachea. The nerve was isolated from the artery using a spinal hook, taking great care not to damage the artery.

To keep the nerve fixed, a pair of blunt-tipped forceps were introduced between the nerve and the rest of the tissue. Then, $1 \mu \mathrm{L}$ of a viral suspension (AAV) was very slowly inoculated using a Hamilton syringe ( $34 \mathrm{G}, 45^{\circ}$ bevel). Finally, the holding forceps were 
removed and the nerve was returned to its initial position. The same procedure was repeated on the nerve of the other side. Once the inoculations finished, the exposed area was closed in layers and the skin incision was closed by means of a simple stitch suture with $4 / 0$ braided silk. Then, anesthesia was reversed by s.c. atipamezole administration (Antisedan, Ecuphar, Spain).

The animals were then administered for 48 hours with analgesic and antibiotic. A possible nerve injury was also monitored by analysis of urination and defecation capacity.

\section{Novel object recognition test}

The Novel object recognition test (NORT) was performed following a protocol previously described (Gomis-González et al., 2021).

On the first day, mice were habituated to the V-shaped maze (V maze) for 9 min. Next day, 2 identical objects (familiar objects) were presented at the end of each corridor of the $\mathrm{V}$-maze and mice were left to explore for $9 \mathrm{~min}$ (familiarization phase). On the last day, $24 \mathrm{~h}$ or $48 \mathrm{~h}$ after the familiarization phase, one of the familiar objects was replaced by a new object (novel object) and mice were placed back in the V-maze to evaluate memory performance (test phase). In this phase, the time spent exploring each of the objects (familiar and novel) was measured to calculate a discrimination index $(\mathrm{DI}=($ Time Novel Object - Time Familiar Object)/(Time Novel Object + Time Familiar Object)), defining exploration as the orientation of the nose toward the object at a distance closer than $1 \mathrm{~cm}$. A higher discrimination index is considered to reflect greater memory retention for the familiar object. Mice that explored $<10 \mathrm{sec}$ both objects during the test session or $<2 \mathrm{sec}$ one of the objects were excluded from the analysis. Drug treatment was administered after the familiarization phase. 


\section{Locomotor activity}

Locomotor activity was assessed for 120 min after acute administration of AM6545. Individual locomotor activity boxes $(9 \times 20 \times 11 \mathrm{~cm})$ (Imetronic) were used in a dim environment. The total activity and the total number of rearings were detected by infrared sensors to detect locomotor activity and infrared plane to detect rearings.

\section{Tissue preparation for immunofluorescence}

Mice were deeply anesthetized by i.p. injection $(0.2 \mathrm{~mL} / 10 \mathrm{~g}$ of body weight $)$ of a mixture of ketamine/xylazine (100 mg/kg and $20 \mathrm{mg} / \mathrm{kg}$, respectively) prior to intracardiac perfusion of cold 4\% paraformaldehyde in $0.1 \mathrm{M}$ phosphate buffer, pH7.5 (PB) delivered with a peristaltic pump at $19 \mathrm{~mL} / \mathrm{min}$ flow for $3 \mathrm{~min}$. Brains were removed and post-fixed overnight at $4{ }^{\circ} \mathrm{C}$ in the same fixative solution. The next day, brains were moved to PB at $4{ }^{\circ} \mathrm{C}$. Coronal brain sections of $30 \mu \mathrm{m}$ were made on a freezing microtome (Leica SM200R) and stored in a 5\% sucrose solution at $4{ }^{\circ} \mathrm{C}$ until use.

\section{Immunofluorescence}

Free-floating brain slices were rinsed in PB and blocked in a solution containing $3 \%$ donkey serum (DS) (Sigma-Aldrich) and $0.3 \%$ Triton X-100 (T) in PB (DS-T-PB) at room temperature for $2 \mathrm{~h}$, and incubated overnight in the same solution with the primary antibody to c-Fos (sc-7202, 1:1000, rabbit, Santa Cruz Biotechnology) at $4{ }^{\circ} \mathrm{C}$. Twenty four hours later, slices were rinsed 3 times in PB and incubated at room temperature with the secondary antibody AlexaFluor-555 donkey anti-rabbit (1:1000, Jackson ImmunoResearch) in DS-T-PB for $2 \mathrm{~h}$. Then, sections were rinsed and mounted onto gelatin-coated slides with Mowiol mounting medium. 


\section{Image acquisition and cell quantification}

Immunostained brain sections were analysed with a $\times 10$ objective using a Leica DMR microscope (Leica Microsystems) equipped with a digital camera Leica DFC 300FX (Leica Microsystems). Seven different brain subregions were analysed: prelimbic cortex (PL), infralimbic cortex (IL), dentate gyrus (DG), Cornu Ammonis 1 (CA1) and 3 (CA3), basal amygdala (BA), central amygdala (CeA) and lateral amygdala (LA). For PL and IL analysis, a 430- $\mu \mathrm{m}$-sided square region of interest (ROI) was delimited for quantification. For the rest of regions, the DAPI signal was used as counterstaining to identify and delimitate each area for quantification. The images were processed using the ImageJ analysis software. c-Fos-positive neurons in each brain area were quantified using the automatic "particle counting" option with a fixed configuration that solely detected cFos-positive cell bodies matching common criteria of size and circularity. A fixed threshold interval was set to distinguish the c-Fos-positive nuclei from the background. In addition, all quantifications were individually checked for homogeneity by an expert observer blind to the experimental conditions. 4-6 representative brain sections of each mouse were quantified, and the average number of c-Fos-positive neurons was calculated for each mouse. The data are expressed as density: the mean number of c-Fos-positive cells per squared mm ( $\mathrm{n}=6$ mice per experimental group). For the c-Fos data, the displayed images were flipped for orientation consistency and transformed to inverted grey scale.

\section{C-Fos based network construction and graph analysis}

Correlation matrices and circos plot were generated using Pearson $r$ values from interregional correlations of c-fos expression data. Pearson $r$ values were converted to Zscores using Fisher's transformation to determine effects of AM6545 on positive and 
negative functional connectivity and compare across experimental groups. All graph analysis was performed in R (v4.0) using the DescTools (v99.40; Andri Signorell et mult. al., 2021) and the ggcorrplot (v0.1.3; Alboukadel Kassambara, 2019) packages.

\section{rsfMR1: Image acquisition}

Magnetic resonance image was performed in the Magnetic Resonance Core Facility (IDIBAPS 7T MRI Unit, Barcelona, Spain). Experiments were conducted on a 7.0T BioSpec 70/30 horizontal animal scanner (Bruker BioSpin, Ettlingen, Germany), equipped with an actively shielded gradient system (400 mT/m, $12 \mathrm{~cm}$ inner diameter). The receiver coil is a surface coil for the mouse brain. Animals were sedated (induction dose of $4 \%$ isoflurane in a mixture of $30 \% \mathrm{O}_{2}$ and $70 \% \mathrm{~N}_{2}$ ) and placed in supine position in a Plexiglas holder with a nose cone for administering anesthetic gases and were fixed using tooth and ear bars and adhesive tape. Eyes were protected from dryness with Siccafluid $2.5 \mathrm{mg} / \mathrm{g}$ ophthalmologic fluid. Once placed in the holder and keeping isoflurane to $1.5 \%$, a subcutaneous bolus $(0.3 \mathrm{mg} / \mathrm{kg})$ of medetomidine (Domtor, Orion Pharma, Spain) was injected. For the next $15 \mathrm{~min}$, the isoflurane dose was progressively decreased until $0.5 \%$. Then, a continuous perfusion of $0.6 \mathrm{mg} / \mathrm{kg} / \mathrm{h}$ medetomidine started and was maintained until the end of the acquisition session. After completion of the imaging session, $1 \mu \mathrm{L} / \mathrm{g}$ of atipamezol per mouse weight (Antisedan, Orion Pharma, Spain) and saline were injected to reverse the sedative effect and compensate fluid loss. Localizer scans were used to ensure accurate position of the head at the isocenter of the magnet. Anatomical T2 RARE images were acquired in coronal orientation with effective $\mathrm{TE}=33 \mathrm{~ms}$ TR $=2.3 \mathrm{~s}$, RARE factor $=8$, voxel size $=0.08 \times 0.08 \mathrm{~mm}^{2}$ and slice thickness $=0.5 \mathrm{~mm}$. rs-fMRI was acquired with an EPI sequence with $\mathrm{TR}=2 \mathrm{~s}, \mathrm{TE}=19.4$, voxel size 
$0.21 \times 0.21 \mathrm{~mm}^{2}$ and slice thickness $0.5 \mathrm{~mm} .420$ volumes were acquired resulting in an acquisition time of $14 \mathrm{~min}$.

\section{rsfMR1: Image analysis and processing}

Two approaches were used to evaluate functional connectivity. On the one hand, wholebrain connectivity was evaluated using global and regional network metrics (Rubinov \& Sporns, 2010) and on the other hand seed-based analysis was performed to evaluate connectivity of posterior brainstem with the rest of the brain. For both approaches, rsfMRI was preprocessed, including slice timing, motion correction by spatial realignment using SPM8, correction of EPI distortion by elastic registration to the T2-weighted volume using ANTs (Avants et al., 2008), detrend, smoothing with a full-width half maximum (FWHM) of $0.6 \mathrm{~mm}$, frequency filtering of the time series between 0.01 and $0.1 \mathrm{~Hz}$ and regression by motion parameters. All these steps were performed using NiTime (http://nipy.org/nitime). Brain parcellation was performed by registration of a mouse brain atlas (Ma et al., 2008) to the T2-RARE acquisition of each subject using ANTs diffeomorphic registration. Region parcellation was then registered from the T2weighted volume to the preprocessed mean rs-fMRI. Whole-brain functional brain network was estimated considering the gray matter regions obtained by parcellation as the network nodes. Connectivity between each pair of regions was estimated as the Fisher-z transform of the correlation between average time series in each region. Network organization was quantified using regional and global graph metrics. Strength, local efficiency and clustering coefficient was computed for every region and whole brain connectivity was quantified by average strength, global and local efficiency and average clustering coefficient (Rubinov \& Sporns, 2010). To perform the seed-based analysis, posterior brainstem containing the nuclei of the solitary tract was manually drawn in the 
images of each animal. Average time series in the seed was computed and correlated with each voxel time series, resulting in a correlation map describing the connectivity of the region of interest with the rest of the brain. Voxel-wise differences between the correlation maps were estimated using fsl-randomize, that performs non-parametric permutation test including family-wise error correction on neuroimaging data (Winkler et al., 2014).

\section{In vivo microdialysis}

Animals were anesthetized with isoflurane $(1.5-2.5 \% \mathrm{v} / \mathrm{v}$ for induction and maintenance) and placed in a Kopf stereotaxic frame. Intracerebral probe (cuprophan membrane of $2 \mathrm{~mm}$ ) was implanted in the hippocampus and fixed to the skull. The target injection site coordinates were as follows: anteroposterior, $-3.40 \mathrm{~mm}$; mediolateral, $\pm 2.60 \mathrm{~mm}$; dorsoventral, $-4.20 \mathrm{~mm}$ relative to bregma (Paxinos and Franklin, 2001). The next day, mice were placed in a plastic bowl and connected to a fraction collection system for freely-moving animals (Raturn, BASi, USA). The input tube of the dialysis probe was connected to a syringe pump (BeeHive and BabyBee, BASi), which delivered a modified cerebrospinal fluid (CSF) containing $\mathrm{NaCl} 148 \mathrm{mM}, \mathrm{KCl} 2.7 \mathrm{mM}, \mathrm{CaCl} 21.2 \mathrm{mM}$ and $\mathrm{MgCl} 20.85 \mathrm{mM}(\mathrm{pH} 7.4)$ to the probe at a rate of $1 \mu \mathrm{L} / \mathrm{min}$. The output tubes from the animals were attached to a refrigerated fraction collector (HoneyComb, BASi). Samples were collected every $35 \mathrm{~min}$ for the analysis of the different neurotransmitters in vials containing $5 \mu \mathrm{L}$ of acetic acid 0.1 M. Eight baseline samples were collected from each animal, but only the last 6 ones were used for subsequent analysis. 
Neurotransmitter concentrations were measured immediately after samples collection by Ultra Performance Liquid Chromatography (UPLC) coupled to an electrochemical detector (Alexys analyser, Antec Leyden, Holland). The mobile phase consisted of 100 $\mathrm{mM}$ phosphoric acid, $100 \mathrm{mM}$ citric acid, $0.1 \mathrm{mM}$ EDTA, 950-1500 mg/L 1octanesulfonic acid (OSA), 5\% v/v acetonitrile; the $\mathrm{pH}$ was adjusted to 6.0 with $50 \%$ $\mathrm{NaOH} / 45 \% \mathrm{KOH}$ solution. The flow rate of the mobile phase was $0.075 \mathrm{~mL} / \mathrm{min}$ and the temperature for the analytical column (Acquity UPLC BEH C18, $1.7 \mu \mathrm{m}, 1 \times 100 \mathrm{~mm}$; Waters, Milford, USA) was $37^{\circ} \mathrm{C}$.

\section{In vivo electrophysiological recording}

Mice were anaesthetized with uretano (1.2 g/kg, i.p.), and placed in the stereotaxic frame with the skull positioned horizontally. A burr hole was drilled and the recording site coordinates were as follows: anteroposterior, $-1.5 \mathrm{~mm}$; mediolateral, $\pm 0.2-1.2 \mathrm{~mm}$; dorsoventral, -2.7-4.0 $\mathrm{mm}$ relative to lambda (Gobbi et al., 2007). The body temperature was maintained at $37^{\circ} \mathrm{C}$ for the entire experiment using a heating pad.

Single-unit extracellular recordings of mouse LC neurons were performed as previously described (Gobbi et al., 2007). The recording electrode was filled with $2 \%$ solution of Pontamine Sky Blue in $0.5 \%$ sodium acetate and broken back to a tip diameter of $1-2$ $\mathrm{mm}$. The electrode was lowered into the brain by means of a hydraulic microdrive (model 640; David Kopf Instruments). LC neurons were identified by standard criteria, which included spontaneous activity displaying a regular rhythm and firing rate between 0.5 and $5 \mathrm{~Hz}$, characteristic spikes with a long-lasting (>2 ms), positive-negative waveform action potentials and the biphasic excitation-inhibition response to pressure applied on contralateral hind paw (paw pinch), as previously described in mice (Gobbi et al., 2007) and rats (Cedarbaum \& Aghajanian, 1978). The extracellular signal from the electrode 
was pre-amplified and amplified later with a high-input impedance amplifier and then monitored on an oscilloscope and on an audio monitor. This activity was processed using computer software (Spike2 software; Cambridge Electronic Design) and firing rate was calculated. Basal firing rate and other electrophysiological parameters were measured for 3 min. Changes in firing rate were expressed as percentages of the basal firing rate (mean firing rate for $3 \mathrm{~min}$ prior to drug injection) and were measured after $35 \mathrm{~min}$ until the end of the experiment. Only 1 cell was studied in each animal when any drug was administered.

\section{Statistical analysis}

Results are reported as mean \pm standard error of the mean (s.e.m). Data analysis were performed using GraphPad Prism software (GraphPad Software). Statistical comparisons were evaluated using unpaired Student's t-test for 2 groups comparisons or two-way ANOVA for multiple comparisons. Subsequent Tukey post hoc was used when required (significant interaction between factors). Comparisons were considered statistically significant when $p<0.05$.

\section{Acknowledgements}

We thank Dulce Real, Marta Linares and Francisco Porrón for expert technical assistance, and the Laboratory of Neuropharmacology-NeuroPhar for helpful discussion.

S.M-T. was the recipient of a predoctoral fellowship (Generalitat de Catalunya) [FIB00531 2016], A.B-M. was supported by predoctoral fellowship (Generalitat de Catalunya) [FI_B0052 2020]. This study was supported by Ministerio de Economía, Innovación y Competitividad (MINECO), Spain (\#RTI2018-099282-B-I00B to A.O., \# PID2020-120029GB-I00 to R.M.; Generalitat de Catalunya, Spain (2017SGR-669 to 
R.M.); Basque Government, Spain (IT-1211-19 to J.J.M); ICREA (Institució Catalana de Recerca i Estudis Avançats, Spain) Academia to A.O. and R.M. Grant "Unidad de Excelencia María de Maeztu", funded by the MINECO (\#MDM-2014-0370). FEDER, European Commission funding is also acknowledged.

This research was supported by PRPSEM Project with ref. RTI2018-099773-B-I00 from (MCIU/FEDER/AEI), the CERCA Program, and the Commission for Universities and Research of the Department of Innovation, Universities, and Enterprise of the Generalitat de Catalunya (SGR2017-648) to JADR. IBEC is the recipients of a Severo Ochoa Award of Excellence from MINECO (CEX2018-000789-S). SMT and AH were supported by Severo Ochoa program at IBEC.

S.M-T. and A.B-M. performed the behavioral, biochemical, histological, confocal and surgery experiments, statistical analyses and graphs and wrote the manuscript. L.G-L. performed bilateral hippocampal cannula implantation in mice. F.R. and B.L. generated and provided the conditional transgenic mice (DBH-CB1KO). A.O-A. performed adrenalectomy in mice. A.H. and JA.dR. designed and performed the chemogenetic experiment. A.H. performed chemogenetic surgeries in the vagus nerve. E.M-M. and G.S. performed and analyzed the rsfMRI experiments and conectomics on c-Fos-based network. JE.O. and JJ.M. performed the microdyalisis in vivo experiments in mice. JA.RO. designed and performed in vivo recordings in mice. R.M. supervised the study; A.O. conceptualized, supervised the study and wrote the manuscript. All authors revised the final version of the manuscript. 


\section{References}

Alboukadel Kassambara. (2019). Visualization of a Correlation Matrix using "ggplot2" ( $\mathrm{R}$ package version 0.1.3).

Andri Signorell et mult. al. (2021). DescTools: Tools for descriptive statistics ( $\mathrm{R}$ package version 0.99.42).

Avants, B. B., Epstein, C. L., Grossman, M., \& Gee, J. C. (2008). Symmetric diffeomorphic image registration with cross-correlation: Evaluating automated labeling of elderly and neurodegenerative brain. Medical Image Analysis, 12(1), 26-41. https://doi.org/10.1016/j.media.2007.06.004

Bacon, T. J., Pickering, A. E., \& Mellor, J. R. (2020). Noradrenaline release from locus coeruleus terminals in the hippocampus enhances excitation-spike coupling in cal pyramidal neurons via $\beta$-adrenoceptors. Cerebral Cortex, 30(12), 6135-6151. https://doi.org/10.1093/cercor/bhaa159

Busquets-Garcia, A., Gomis-González, M., Salgado-Mendialdúa, V., Galera-López, L., Puighermanal, E., Martín-García, E., Maldonado, R., \& Ozaita, A. (2018). Hippocampal Protein Kinase C Signaling Mediates the Short-Term Memory Impairment Induced by Delta9-Tetrahydrocannabinol. Neuropsychopharmacology, 43(5), 1021-1031. https://doi.org/10.1038/npp.2017.175

Busquets-Garcia, A., Gomis-González, M., Srivastava, R. K., Cutando, L., OrtegaAlvaro, A., Ruehle, S., Remmers, F., Bindila, L., Bellocchio, L., Marsicano, G., Lutz, B., Maldonado, R., \& Ozaita, A. (2016). Peripheral and central CB1 cannabinoid receptors control stress-induced impairment of memory consolidation. Proceedings of the National Academy of Sciences of the United States of America, 113(35), 9904-9909. https://doi.org/10.1073/pnas.1525066113

Busquets-Garcia, A., Puighermanal, E., Pastor, A., de la Torre, R., Maldonado, R., \& Ozaita, A. (2011). Differential role of anandamide and 2-arachidonoylglycerol in memory and anxiety-like responses. Biological Psychiatry, 70(5), 479-486. https://doi.org/10.1016/j.biopsych.2011.04.022

Castillo, P. E., Younts, T. J., Chávez, A. E., \& Hashimotodani, Y. (2012). Endocannabinoid Signaling and Synaptic Function. In Neuron (Vol. 76, Issue 1, pp. 70-81). Neuron. https://doi.org/10.1016/j.neuron.2012.09.020

Cedarbaum, J. M., \& Aghajanian, G. K. (1978). Afferent projections to the rat locus coeruleus as determined by a retrograde tracing technique. The Journal of Comparative Neurology, 178(1), 1-15. https://doi.org/10.1002/cne.901780102

Cohen, S. J., \& Stackman, R. W. (2015). Assessing rodent hippocampal involvement in the novel object recognition task. A review. Behavioural Brain Research, 285, 105-117. https://doi.org/10.1016/j.bbr.2014.08.002

De Quervain, D., Schwabe, L., \& Roozendaal, B. (2016). Stress, glucocorticoids and memory: Implications for treating fear-related disorders. In Nature Reviews Neuroscience (Vol. 18, Issue 1, pp. 7-19). Nature Publishing Group. https://doi.org/10.1038/nrn.2016.155

Dornelles, A., de Lima, M. N. M., Grazziotin, M., Presti-Torres, J., Garcia, V. A., Scalco, F. S., Roesler, R., \& Schröder, N. (2007). Adrenergic enhancement of consolidation of object recognition memory. Neurobiology of Learning and Memory, 88(1), 137-142. https://doi.org/10.1016/j.nlm.2007.01.005

Ghacibeh, G. A., Shenker, J. I., Shenal, B., Uthman, B. M., \& Heilman, K. M. (2006). The influence of vagus nerve stimulation on memory. Cognitive and Behavioral Neurology, 19(3), 119-122. https://doi.org/10.1097/01.wnn.0000213908.34278.7d Gobbi, G., Cassano, T., Radja, F., Morgese, M. G., Cuomo, V., Santarelli, L., Hen, R., 
\& Blier, P. (2007). Neurokinin 1 receptor antagonism requires norepinephrine to increase serotonin function. European Neuropsychopharmacology, 17(5), 328338. https://doi.org/10.1016/j.euroneuro.2006.07.004

Gold, P. E., \& Korol, D. L. (2012). Making memories matter. Frontiers in Integrative Neuroscience, 6(NOV). https://doi.org/10.3389/fnint.2012.00116

Gomis-González, M., Galera-López, L., Ten-Blanco, M., Busquets-Garcia, A., Cox, T., Maldonado, R., \& Ozaita, A. (2021). Protein Kinase C-Gamma Knockout Mice Show Impaired Hippocampal Short-Term Memory While Preserved Long-Term Memory. Molecular Neurobiology, 58(2), 617-630. https://doi.org/10.1007/s12035-020-02135-6

Hagena, H., Hansen, N., \& Manahan-Vaughan, D. (2016). $\beta$-Adrenergic Control of Hippocampal Function: Subserving the Choreography of Synaptic Information Storage and Memory. Cerebral Cortex, 26(4), 1349-1364. https://doi.org/10.1093/cercor/bhv330

Hansen, \& Manahan-Vaughan, D. (2015). Locus Coeruleus Stimulation Facilitates Long-Term Depression in the Dentate Gyrus That Requires Activation of Adrenergic Receptors. Cerebral Cortex, 25(7), 1889-1896. https://doi.org/10.1093/cercor/bht429

Hansen, N. (2017). The Longevity of Hippocampus-Dependent Memory Is Orchestrated by the Locus Coeruleus-Noradrenergic System. Neural Plasticity, 2017, 1-9. https://doi.org/10.1155/2017/2727602

Hillard, C. J. (2015). Endocannabinoids and the Endocrine System in Health and Disease. In Handbook of experimental pharmacology (Vol. 231, pp. 317-339). https://doi.org/10.1007/978-3-319-20825-1_11

Jacob, W., Marsch, R., Marsicano, G., Lutz, B., \& Wotjak, C. T. (2012). Cannabinoid $\mathrm{CB} 1$ receptor deficiency increases contextual fear memory under highly aversive conditions and long-term potentiation in vivo. Neurobiology of Learning and Memory, 98(1), 47-55. https://doi.org/10.1016/j.nlm.2012.04.008

Kandel, E. R., Dudai, Y., \& Mayford, M. R. (2014). The molecular and systems biology of memory. In Cell (Vol. 157, Issue 1, pp. 163-186). Cell Press. https://doi.org/10.1016/j.cell.2014.03.001

Kano, M., Ohno-Shosaku, T., Hashimotodani, Y., Uchigashima, M., \& Watanabe, M. (2009). Endocannabinoid-Mediated Control of Synaptic Transmission.

Physiological Reviews, 89(1), 309-380.

https://doi.org/10.1152/physrev.00019.2008

Kemp, A., \& Manahan-Vaughan, D. (2012). Passive spatial perception facilitates the expression of persistent hippocampal long-term depression. Cerebral Cortex (New York, N.Y. : 1991), 22(7), 1614-1621. https://doi.org/10.1093/cercor/bhr233

Kemp, \& Manahan-Vaughan, D. (2008). -Adrenoreceptors Comprise a Critical Element in Learning-Facilitated Long-Term Plasticity. Cerebral Cortex, 18(6), 1326-1334. https://doi.org/10.1093/cercor/bhm164

Kilkenny, C., Browne, W., Cuthill, I. C., Emerson, M., Altman, D. G., \& NC3Rs Reporting Guidelines Working Group. (2010). Animal research: Reporting in vivo experiments: The ARRIVE guidelines. British Journal of Pharmacology, 160(7), 1577-1579. https://doi.org/10.1111/j.1476-5381.2010.00872.x

Kitamura, T., Ogawa, S. K., Roy, D. S., Okuyama, T., Morrissey, M. D., Smith, L. M., Redondo, R. L., \& Tonegawa, S. (2017). Engrams and circuits crucial for systems consolidation of a memory. Science (New York, N.Y.), 356(6333), 73-78. https://doi.org/10.1126/science.aam6808

Lee, H. J., Berger, S. Y., Stiedl, O., Spiess, J., \& Kim, J. J. (2001). Post-training 
injections of catecholaminergic drugs do not modulate fear conditioning in rats and mice. Neuroscience Letters, 303(2), 123-126. https://doi.org/10.1016/S03043940(01)01733-5

Lichtman, A. H. (2000). SR 141716A enhances spatial memory as assessed in a radialarm maze task in rats. European Journal of Pharmacology, 404(1-2), 175-179.

Ma, Y., Smith, D., Hof, P. R., Foerster, B., Hamilton, S., Blackband, S. J., Yu, M., \& Benveniste, H. (2008). In vivo 3D digital atlas database of the adult C57BL/6J mouse brain by magnetic resonance microscopy. Frontiers in Neuroanatomy, 2(APR). https://doi.org/10.3389/neuro.05.001.2008

Maccarrone, M., Bab, I., Bíró, T., Cabral, G. A., Dey, S. K., Di Marzo, V., Konje, J. C., Kunos, G., Mechoulam, R., Pacher, P., Sharkey, K. A., \& Zimmer, A. (2015). Endocannabinoid signaling at the periphery: 50 years after THC. Trends in Pharmacological Sciences, 36(5), 277-296.

https://doi.org/10.1016/j.tips.2015.02.008

Maccarrone, M., Valverde, O., Barbaccia, M. L., Castañé, A., Maldonado, R., Ledent, C., Parmentier, M., \& Finazzi-Agrò, A. (2002). Age-related changes of anandamide metabolism in CB1 cannabinoid receptor knockout mice: correlation with behaviour. The European Journal of Neuroscience, 15(7), 1178-1186.

McIntyre, C. K., McGaugh, J. L., \& Williams, C. L. (2012). Interacting brain systems modulate memory consolidation. Neuroscience \& Biobehavioral Reviews, 36(7), 1750-1762. https://doi.org/10.1016/j.neubiorev.2011.11.001

McReynolds, J. R., Christianson, J. P., Blacktop, J. M., \& Mantsch, J. R. (2018). What does the Fos say? Using Fos-based approaches to understand the contribution of stress to substance use disorders. In Neurobiology of Stress (Vol. 9, pp. 271-285). Elsevier Inc. https://doi.org/10.1016/j.ynstr.2018.05.004

Miyashita, T., \& Williams, C. (2006). Epinephrine administration increases neural impulses propagated along the vagus nerve: Role of peripheral $\beta$-adrenergic receptors. Neurobiology of Learning and Memory, 85(2), 116-124. https://doi.org/10.1016/j.nlm.2005.08.013

Morris, R. G. M. (2006). Elements of a neurobiological theory of hippocampal function: the role of synaptic plasticity, synaptic tagging and schemas. The European Journal of Neuroscience, 23(11), 2829-2846. https://doi.org/10.1111/j.14609568.2006.04888.x

Niyuhire, F., Varvel, S. A., Martin, B. R., \& Lichtman, A. H. (2007). Exposure to Marijuana Smoke Impairs Memory Retrieval in Mice. Journal of Pharmacology and Experimental Therapeutics, 322(3), 1067-1075. https://doi.org/10.1124/jpet.107.119594

Pacher, P., Bátkai, S., \& Kunos, G. (2006). The Endocannabinoid System as an Emerging Target of Pharmacotherapy. Pharmacological Reviews, 58(3), 389-462. https://doi.org/10.1124/pr.58.3.2

Paxinos and Franklin. (2001). The Mouse Brain in Stereotaxic Coordinates. Elsevier.

Puighermanal, E., Busquets-Garcia, A., Maldonado, R., \& Ozaita, A. (2012). Cellular and intracellular mechanisms involved in the cognitive impairment of cannabinoids. Philosophical Transactions of the Royal Society of London. Series B, Biological Sciences, 367(1607), 3254-3263. https://doi.org/10.1098/rstb.2011.0384

Puighermanal, E., Marsicano, G., Busquets-Garcia, A., Lutz, B., Maldonado, R., \& Ozaita, A. (2009). Cannabinoid modulation of hippocampal long-term memory is mediated by mTOR signaling. Nature Neuroscience, 12(9), 1152-1158. https://doi.org/10.1038/nn.2369 
Reibaud, M., Obinu, M. C., Ledent, C., Parmentier, M., Böhme, G. A., \& Imperato, A. (1999). Enhancement of memory in cannabinoid CB1 receptor knock-out mice. European Journal of Pharmacology, 379(1), R1-2.

Roberts, C. J., \& Hillard, C. J. (2020). Peripherally restricted cannabinoid type 1 receptor (CB1R) antagonist, AM6545, potentiates stress-induced hypothalamicpituitary-adrenal axis activation via a non-CB1R mechanism. In Endocrine (Vol. 72, Issue 1). Springer. https://doi.org/10.1007/s12020-020-02549-1

Roozendaal, B., \& McGaugh, J. L. (2011). Memory modulation. Behavioral Neuroscience, 125(6), 797-824. https://doi.org/10.1037/a0026187

Rubinov, M., \& Sporns, O. (2010). Complex network measures of brain connectivity: Uses and interpretations. NeuroImage, 52(3), 1059-1069.

https://doi.org/10.1016/j.neuroimage.2009.10.003

Sala-Llonch, R., Peña-Gómez, C., Arenaza-Urquijo, E. M., Vidal-Piñeiro, D., Bargalló, N., Junqué, C., \& Bartrés-Faz, D. (2012). Brain connectivity during resting state and subsequent working memory task predicts behavioural performance. Cortex, 48(9), 1187-1196. https://doi.org/10.1016/j.cortex.2011.07.006

Sara, S. J. (2009). The locus coeruleus and noradrenergic modulation of cognition. Nature Reviews Neuroscience, 10(3), 211-223. https://doi.org/10.1038/nrn2573

Silva, B. A., Burns, A. M., \& Gräff, J. (2019). A cFos activation map of remote fear memory attenuation. Psychopharmacology, 236(1), 369-381. https://doi.org/10.1007/s00213-018-5000-y

Takahashi, R. N., Pamplona, F. A., \& Fernandes, M. S. (2005). The cannabinoid antagonist SR141716A facilitates memory acquisition and consolidation in the mouse elevated T-maze. Neuroscience Letters, 380(3), 270-275. https://doi.org/10.1016/j.neulet.2005.01.049

Talley, C. E., Kahn, S., Alexander, L. J., \& Gold, P. E. (2000). Epinephrine fails to enhance performance of food-deprived rats on a delayed spontaneous alternation task. Neurobiology of Learning and Memory, 73(1), 79-86. https://doi.org/10.1006/nlme.1999.3920

Tam, J., Vemuri, V. K., Liu, J., Bátkai, S., Mukhopadhyay, B., Godlewski, G., OseiHyiaman, D., Ohnuma, S., Ambudkar, S. V., Pickel, J., Makriyannis, A., \& Kunos, G. (2010). Peripheral CB1 cannabinoid receptor blockade improves cardiometabolic risk in mouse models of obesity. Journal of Clinical Investigation, 120(8), 2953-2966. https://doi.org/10.1172/JCI42551

Tanimizu, T., Kono, K., \& Kida, S. (2017). Brain networks activated to form object recognition memory. Brain Research Bulletin. https://doi.org/10.1016/j.brainresbull.2017.05.017

Vázquez-Oliver, A., Brambilla-Pisoni, C., Domingo-Gainza, M., Maldonado, R., Ivorra, A., \& Ozaita, A. (2020). Auricular transcutaneous vagus nerve stimulation improves memory persistence in naïve mice and in an intellectual disability mouse model. Brain Stimulation, 13(2), 494-498. https://doi.org/10.1016/j.brs.2019.12.024

Wade, M. R., Degroot, A., \& Nomikos, G. G. (2006). Cannabinoid CB1 receptor antagonism modulates plasma corticosterone in rodents. European Journal of Pharmacology, 551(1-3), 162-167. https://doi.org/10.1016/j.ejphar.2006.08.083

Winkler, A. M., Ridgway, G. R., Webster, M. A., Smith, S. M., \& Nichols, T. E. (2014). Permutation inference for the general linear model. NeuroImage, 92(100), 381-397. https://doi.org/10.1016/j.neuroimage.2014.01.060

Wolff, M. C., \& Leander, J. D. (2003). SR141716A, a cannabinoid CB1 receptor antagonist, improves memory in a delayed radial maze task. European Journal of 
Pharmacology, 477(3), 213-217.

Yang, C., Liu, J.-F., Chai, B.-S., Fang, Q., Chai, N., Zhao, L.-Y., Xue, Y.-X., Luo, Y.X., Jian, M., Han, Y., Shi, H.-S., Lu, L., Wu, P., \& Wang, J.-S. (2013). Stress within a restricted time window selectively affects the persistence of long-term memory. PloS One, 8(3), e59075. https://doi.org/10.1371/journal.pone.0059075

Yonelinas, A. P., Ranganath, C., Ekstrom, A. D., \& Wiltgen, B. J. (2019). A contextual binding theory of episodic memory: systems consolidation reconsidered. In Nature Reviews Neuroscience (Vol. 20, Issue 6, pp. 364-375). Nature Publishing Group. https://doi.org/10.1038/s41583-019-0150-4

Zanettini, C., Panlilio, L. V, Alicki, M., Goldberg, S. R., Haller, J., \& Yasar, S. (2011). Effects of endocannabinoid system modulation on cognitive and emotional behavior. Frontiers in Behavioral Neuroscience, 5, 57. https://doi.org/10.3389/fnbeh.2011.00057

Zhou, M., Bakker, E. H. M., Velzing, E. H., Berger, S., Oitzl, M., Joëls, M., \& Krugers, H. J. (2010). Both mineralocorticoid and glucocorticoid receptors regulate emotional memory in mice. Neurobiology of Learning and Memory, 94(4), 530537. https://doi.org/10.1016/j.nlm.2010.09.005

Zimmer, A., Zimmer, A. M., Hohmann, A. G., Herkenham, M., \& Bonner, T. I. (1999). Increased mortality, hypoactivity, and hypoalgesia in cannabinoid CB1 receptor knockout mice. Proceedings of the National Academy of Sciences of the United States of America, 96(10), 5780-5785. https://doi.org/10.1073/pnas.96.10.5780 


\section{Figures}

a

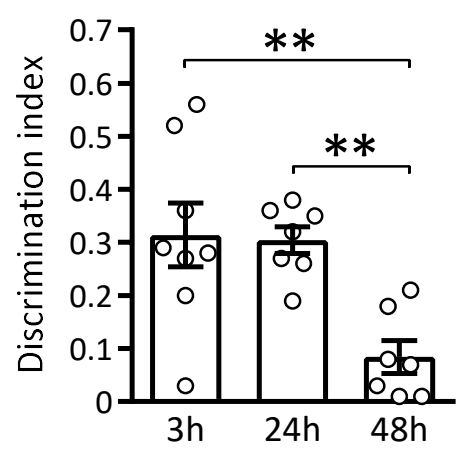

C

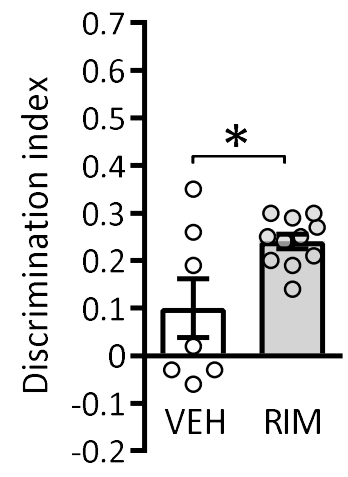

b

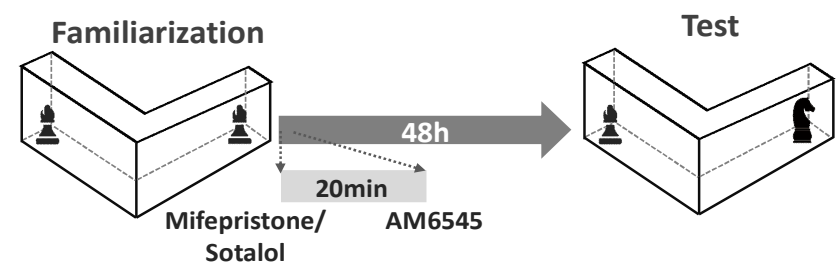

d

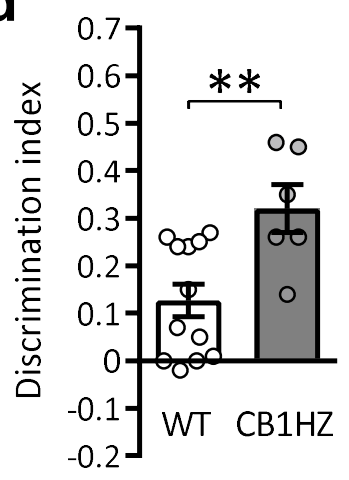

e

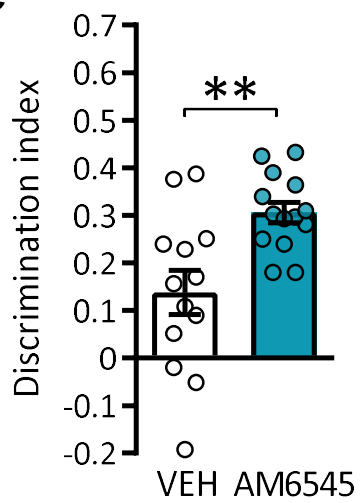

Fig. 1. Pharmacological or genetic inhibition of CB1R improves memory persistence in the novel object-recognition test. a Discrimination index values obtained at $3 \mathrm{~h}, 24 \mathrm{~h}$ and $48 \mathrm{~h}$ after the familiarization phase $(\mathrm{n}=5-8)$. b Schematic representation of pretreatment and treatment after the familiarization phase. c-e Discrimination index values in NORT at $48 \mathrm{~h} \mathbf{c}$ after acute post-familiarization treatment with vehicle (VEH) or rimonabant (RIM) $(1 \mathrm{mg} / \mathrm{kg})(\mathrm{n}=7-11), \mathbf{d}$ in CB1HZ and WT mice $(\mathrm{n}=6-8)$, e after acute post-familiarization treatment with vehicle (VEH) or AM6545 $(1 \mathrm{mg} / \mathrm{kg})(\mathrm{n}=7$ 8). Data are expressed as mean \pm s.e.m. ${ }^{*} p<0.05,{ }^{* *} p<0.01$ by one-way ANOVA test followed by Tukey post hoc or Student's t-test. 
a

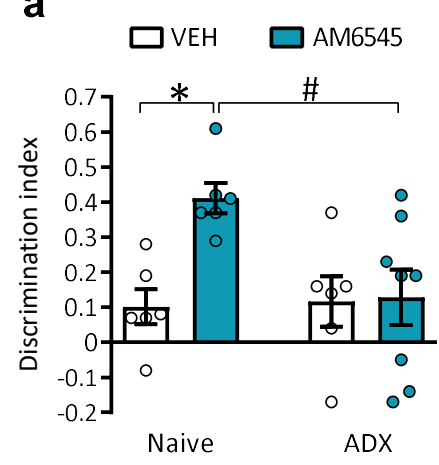

C

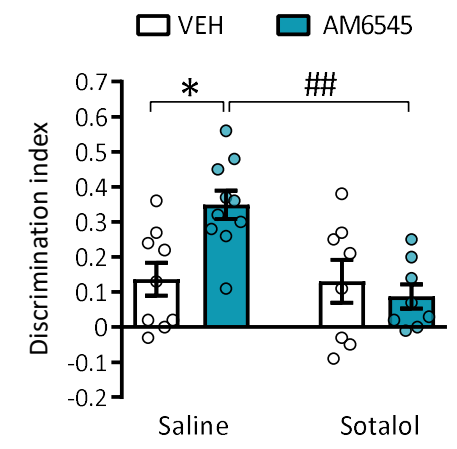

e $\square W T \square D B H-C B 1 K O$

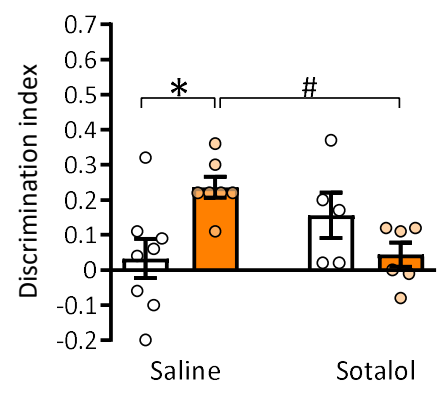

b

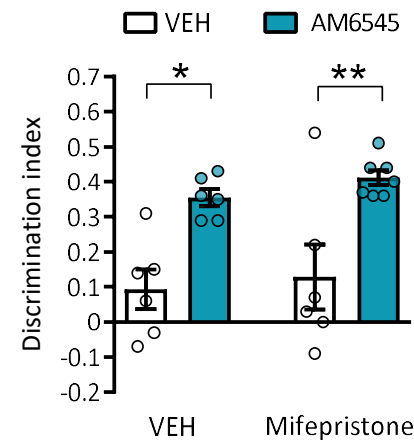

d

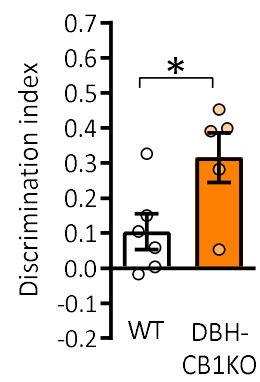

f

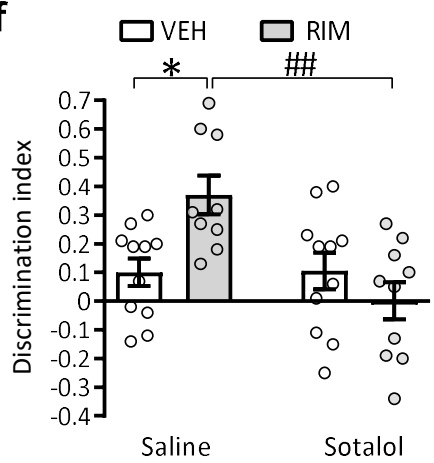

Fig. 2. AM6545 enhances novel object-recognition memory through a peripheral $\beta$ adrenergic mechanism. a Discrimination index values obtained in the NORT performed at $48 \mathrm{~h}$ of adrenalectomized (ADX) or naive mice treated with vehicle (VEH) or AM6545 $(1 \mathrm{mg} / \mathrm{kg})(\mathrm{n}=6-8) . \mathbf{b}$, c Discrimination index values obtained in the NORT performed at $48 \mathrm{~h}$ of mice treated with vehicle $(\mathrm{VEH})$ or AM6545 $(1 \mathrm{mg} / \mathrm{kg})$ after pre-treatment with b vehicle $(\mathrm{VEH})$ or mifepristone $(50 \mathrm{mg} / \mathrm{kg})(\mathrm{n}=6-7)$ and $\mathbf{c}$ saline or sotalol $(10 \mathrm{mg} / \mathrm{kg})$ $(\mathrm{n}=8-10)$. $\mathbf{d}$ Discrimination index values in WT or DBH-CB1KO mice in NORT at 48 $\mathrm{h}(\mathrm{n}=5-6)$ e and after saline or sotalol $(10 \mathrm{mg} / \mathrm{kg})$ treatment $(\mathrm{n}=6-8) \mathbf{f}$ Discrimination index values for mice pre-treated with saline or sotalol $(10 \mathrm{mg} / \mathrm{kg})$ prior to rimonabant (RIM) $(1 \mathrm{mg} / \mathrm{kg})$ or vehicle $(\mathrm{VEH})$ in the NORT at $48 \mathrm{~h}(\mathrm{n}=9-11)$. Data are expressed as mean \pm s.e.m. ${ }^{*} \mathrm{p}<0.05,{ }^{* *} \mathrm{p}<0.01$ (treatment effect) ${ }^{\#} \mathrm{p}<0.05,{ }^{\# \#} \mathrm{p}<0.01$ (pretreatment effect) by two-way ANOVA test followed by Tukey post hoc. 
a

\section{VEH - AM6545}

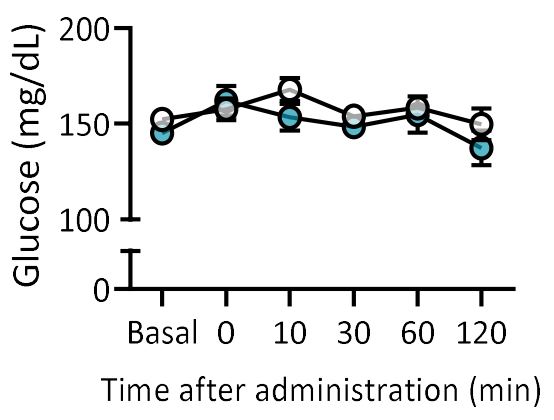

b

ZOCNO-VEH

Q 2 CNO-AM6545

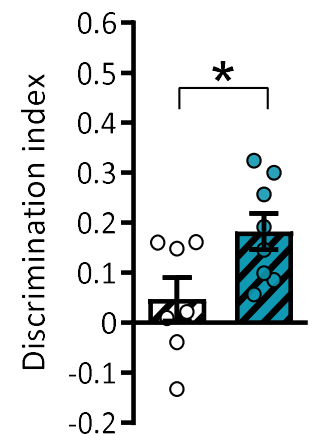

AAV-mCherry
C
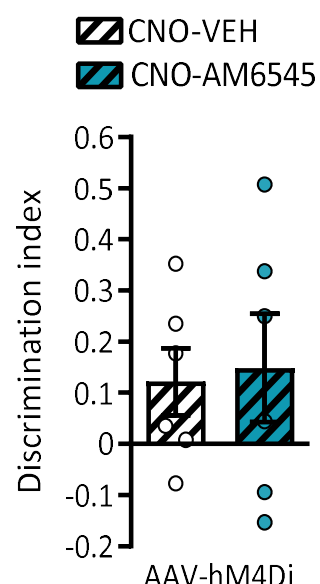

Fig. 3. AM6545 memory improvement depends on vagus nerve activation. a Glucose blood levels for $120 \mathrm{~min}$ after acute vehicle (VEH) or AM6545 $(1 \mathrm{mg} / \mathrm{kg})$ administration in mice $(\mathrm{n}=6-7) \mathbf{b , c}$ Discrimination index values in NORT $48 \mathrm{~h}$ after acute vehicle $(\mathrm{VEH})$ or AM6545 $(1 \mathrm{mg} / \mathrm{kg})$ and CNO $(3 \mathrm{mg} / \mathrm{kg})$ administration in mice infected with b AAV5-mCherry-control or c AAV5-mCherry-hM4Di in the vagus nerve $(n=6-7)$. Data are expressed as mean \pm s.e.m. $* p<0.05$ by Student's $t$ test or two-way repeated measures ANOVA test. 
a Vehicle

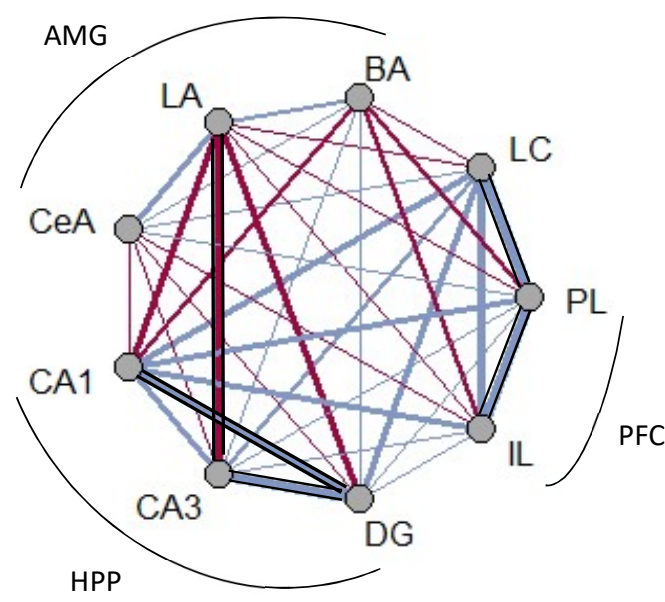

b AM6545

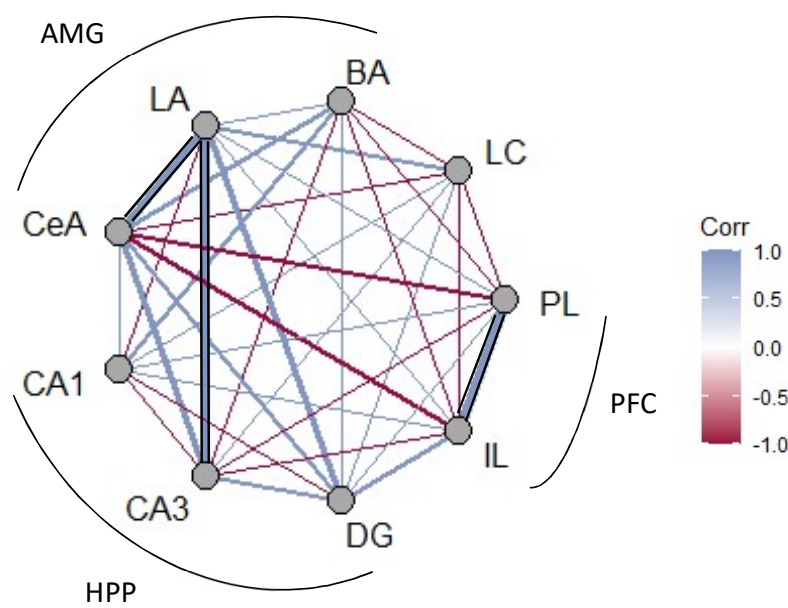

C

d
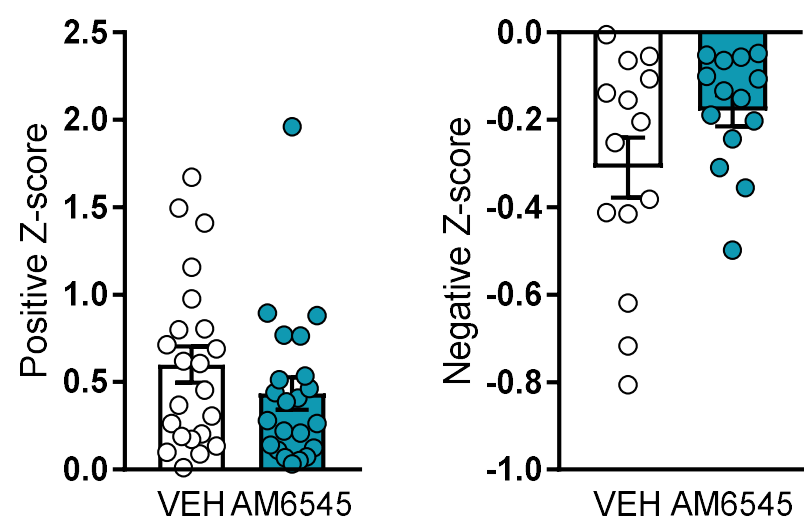

Fig. 4. Circos plot and z-score analysis of c-Fos expression correlation analysis in different brain regions after acute $\mathbf{A M 6 5 4 5}$ administration. Network graphs of c-Fos expression based on the Pearson coefficient in a vehicle and b AM6545 (1 mg/kg) between different brain areas, including prelimbic cortex (PL), infralimbic cortex (IL), cornu ammonis 1 (CA1), dentate gyrus (DG), cornu ammonis 3 (CA3), locus coeruleus (LC), basal amygdala (BA), central amygdala (CeA) and lateral amygdala (LA). Significant correlations are depicted with black lines. c,d Z-score values of the c positive and $\mathbf{d}$ negative Pearson $r$ correlation coefficients between the different brain regions analysed. 
a

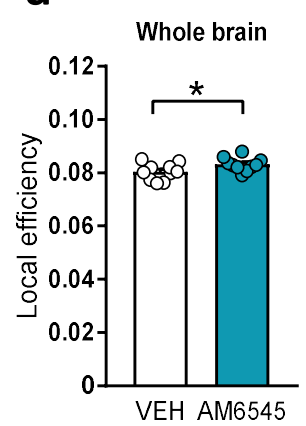

b

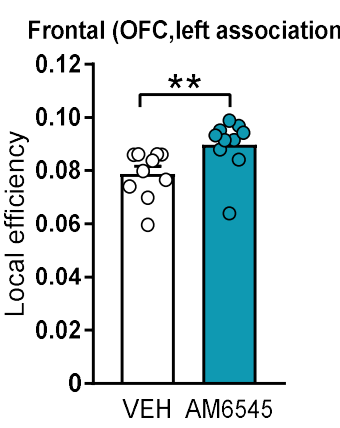

C

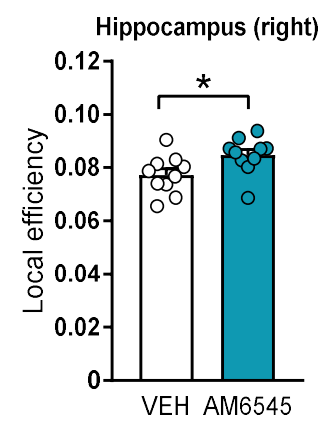

d

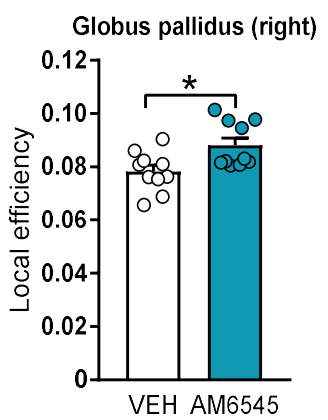

\section{e}

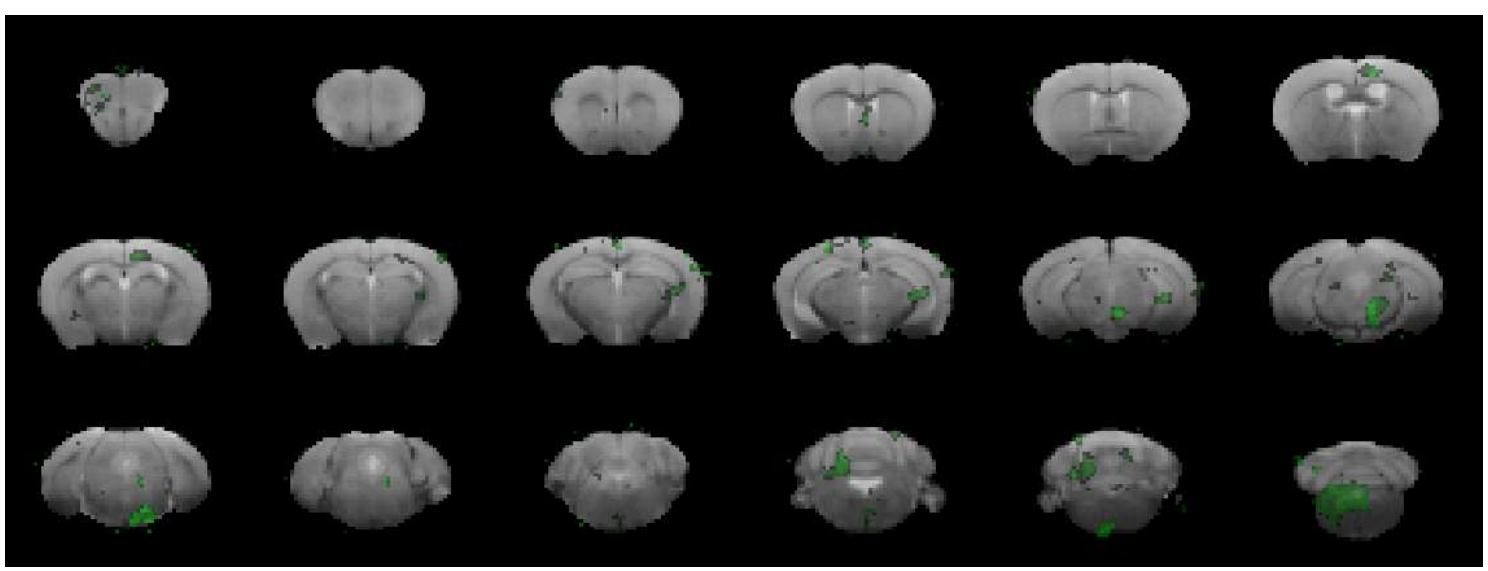

Fig. 5. Effects of AM6545 administration on brain functional connectivity by resting state functional magnetic resonance imaging (rsfMRI). a Whole brain local efficiency and $\mathbf{b}-\mathbf{d}$ nodal efficiencies of $\mathbf{b}$ left frontal cortex, $\mathbf{c}$ right hippocampus and $\mathbf{d}$ right globus pallidus. e Statistical map showing significant differences between connectivity of posterior brainstem with the rest of the brain in vehicle and AM6545 treated animals (comparison vehicle $>$ AM6545; $p<0.01$ ). Data are expressed as mean \pm s.e.m. ${ }^{*} \mathrm{p}<0.05$, $* * \mathrm{p}<0.01$ by Kruskal-Wallis test. 
a

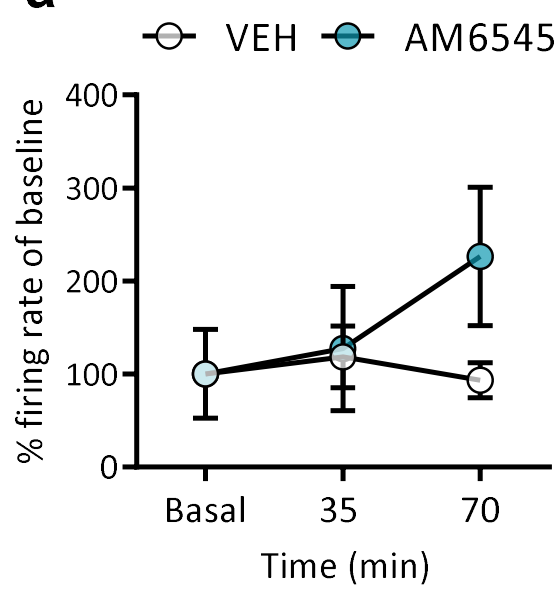

C
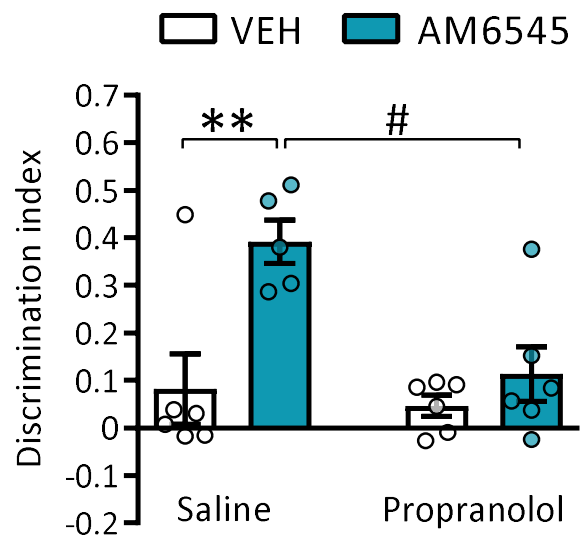
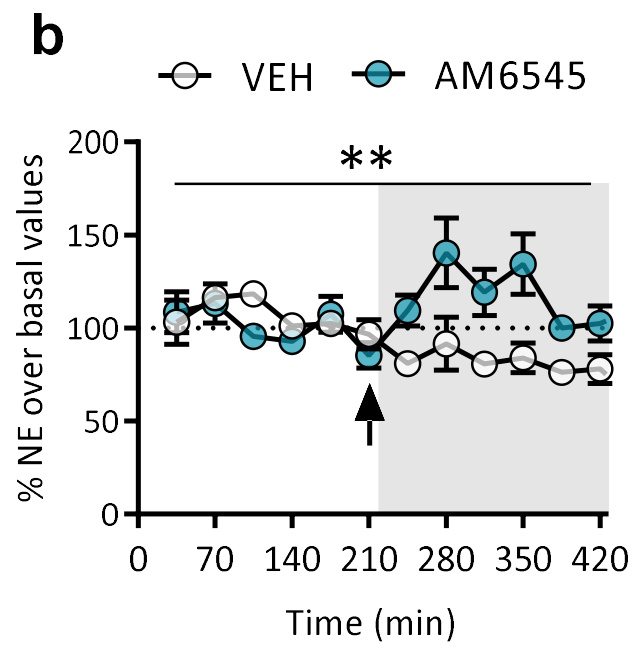

d

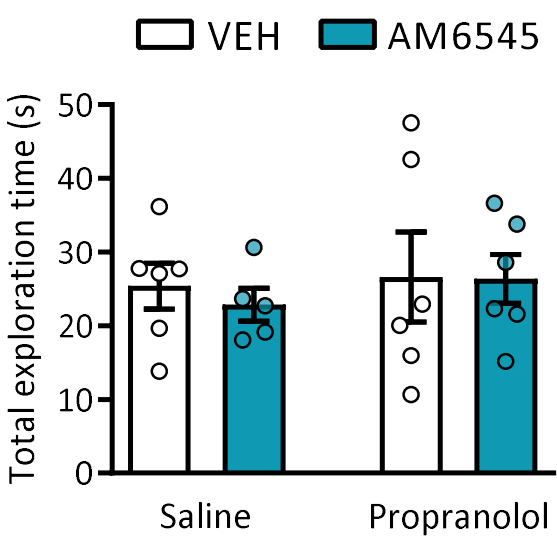

Fig. 6. Acute AM6545 treatment increases central noradrenergic activity. a Percentage of mean firing rate in the LC after acute vehicle (VEH) or AM6545 (1 mg/kg) administration respect to baseline values $(n=2-3)$ b Percentage of extracellular norepinephrine (NE) levels in the hippocampus after acute vehicle (VEH) or AM6545 (1 $\mathrm{mg} / \mathrm{kg}$ ) administration relative to baseline values $(\mathrm{n}=6-7)$. The arrow indicates the time of administration. $\mathbf{c}$ Discrimination index values and $\mathbf{d}$ total exploration time obtained in the NORT performed at $48 \mathrm{~h}$ mice treated with AM6545 $(1 \mathrm{mg} / \mathrm{kg})$ or vehicle (VEH) after bilateral intrahippocampal injection of saline or propranolol $(1 \mu \mathrm{g} / \mu \mathrm{l} 0.5 \mu \mathrm{L}$ per side) (n $=5-6$ ). Data are expressed as mean \pm s.e.m. For microdialysis $* * p<0.01$ two-way repeated measures ANOVA test followed by Tukey post hoc was performed. For NORT $* * \mathrm{p}<0.01$ (treatment effect) ${ }^{\#} \mathrm{p}<0.05$ (pre-treatment effect) by two-way ANOVA test followed by Tukey post hoc. For the firing rate at locus coeruleus statistical significance was calculated by two-way repeated measures ANOVA test. 
a

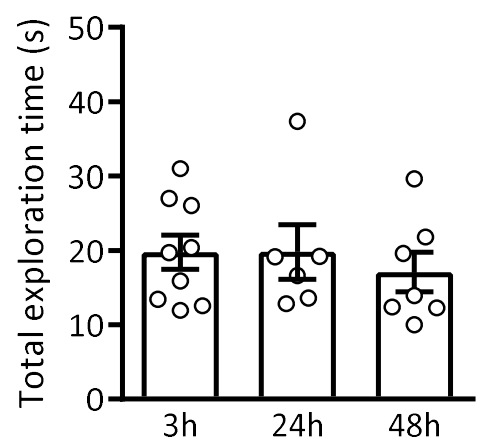

C

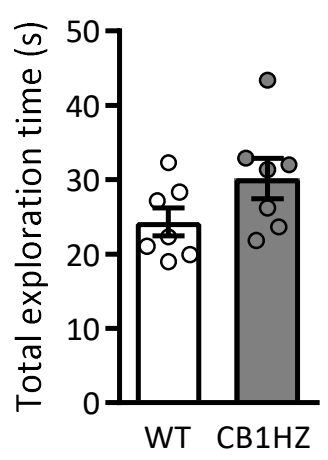

b

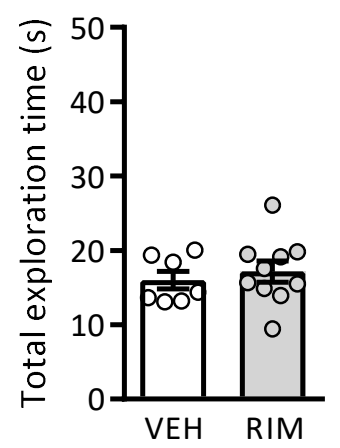

d

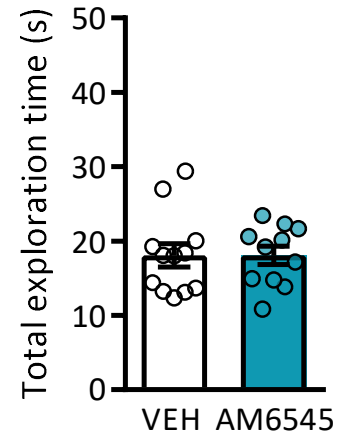

Supplementary Fig. 1. Total exploration times in NORT after pharmacological or genetic CB1R inhibition. a Total exploration times obtained at $3 \mathrm{~h}, 24 \mathrm{~h}$ and $48 \mathrm{~h}$ after the familiarization phase $(\mathrm{n}=5-8) \mathbf{b}-\mathbf{d}$ Total exploration times in NORT at $48 \mathrm{~h} \mathbf{b}$ after acute post-familiarization treatment with vehicle (VEH) or rimonabant (RIM) $(1 \mathrm{mg} / \mathrm{kg}$ ) $(\mathrm{n}=7-11) \mathbf{c}$ in CB1HZ and WT mice $(\mathrm{n}=6-8) \mathbf{d}$ after acute post-familiarization treatment with vehicle (VEH) or AM6545 $(1 \mathrm{mg} / \mathrm{kg})(\mathrm{n}=7-8)$. Data are expressed as mean \pm s.e.m. Statistical significance was calculated by one-way ANOVA test or Student's t test. 
a

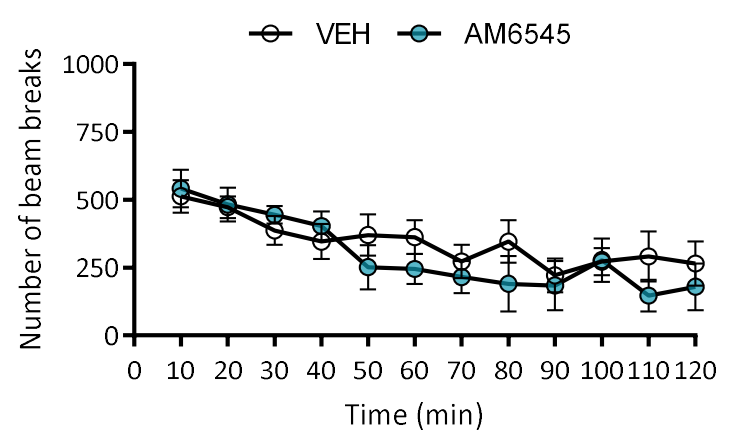

b

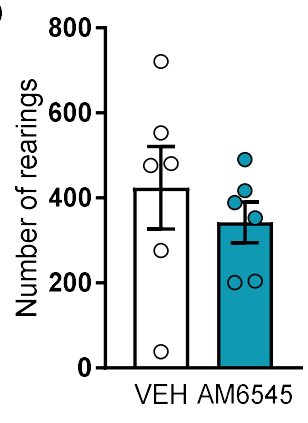

Supplementary Fig. 2. Locomotor activity after acute AM6545 administration. a Total activity and $\mathbf{b}$ total number of rerarings performed in locomotor activity boxes for $120 \mathrm{~min}$ by mice treated with vehicle $(\mathrm{VEH})$ or AM6545 $(1 \mathrm{mg} / \mathrm{kg})(\mathrm{n}=6)$. Data are expressed as mean \pm s.e.m. Statistical significance was calculated by two-way repeated measures ANOVA test or Student's t test. 
a

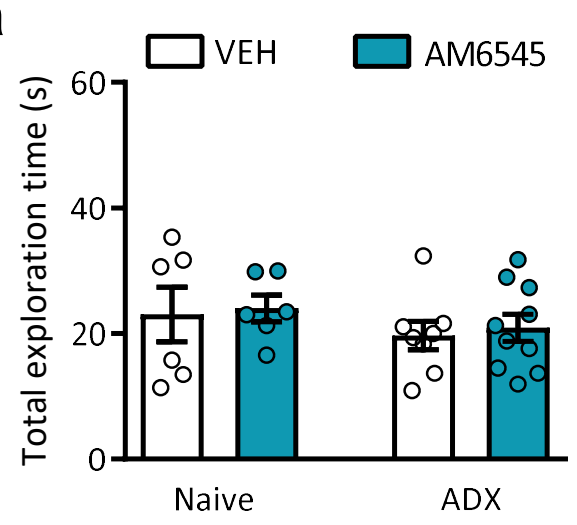

C

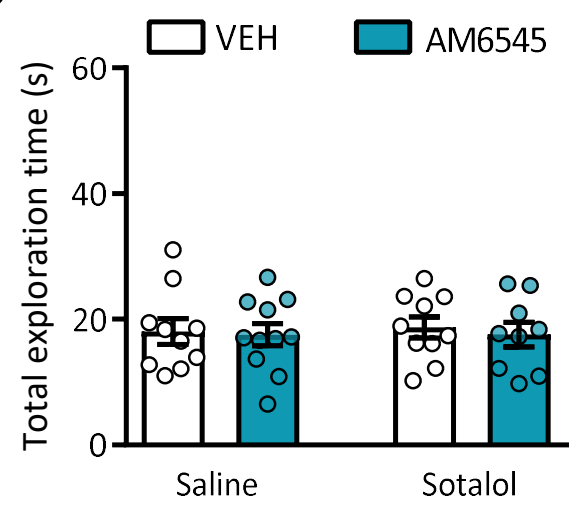

e

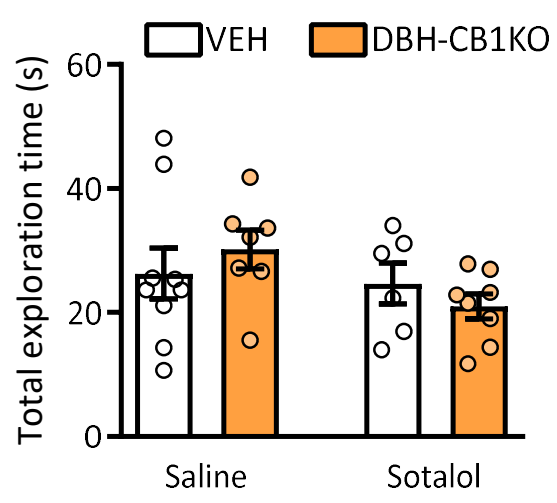

b

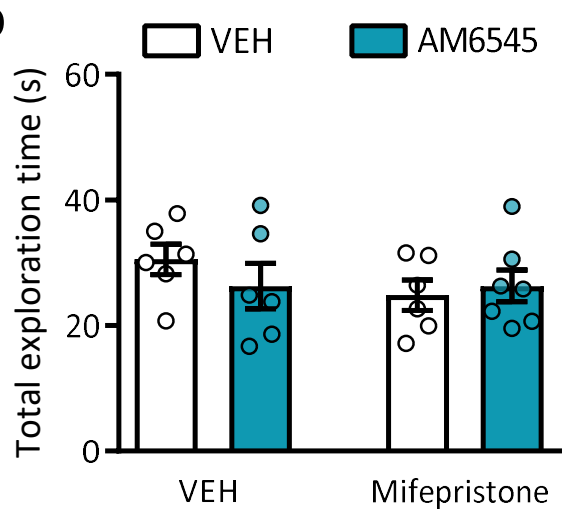

d

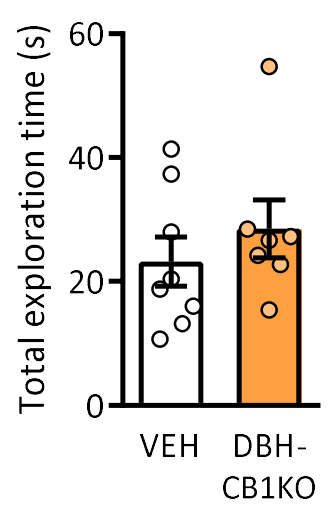

f

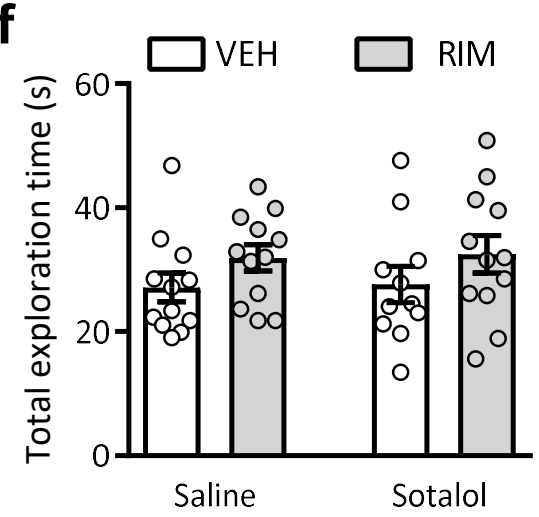

Supplementary Fig. 3. Total exploration times of systemic or peripheral CB1R blockade are not affected. a-f Total exploration times obtained in the NORT performed at $48 \mathrm{~h}$ of a adrenalectomized (ADX) or naive mice treated with vehicle (VEH) or AM6545 $(1 \mathrm{mg} / \mathrm{kg})(\mathrm{n}=6-8), \mathbf{b}, \mathbf{c}$ mice treated with vehicle (VEH) or AM6545 $(1 \mathrm{mg} / \mathrm{kg})$ after pre-treatment with $\mathbf{b}$ vehicle (VEH) or mifepristone $(50 \mathrm{mg} / \mathrm{kg})(\mathrm{n}=6-7)$ and $\mathbf{c}$ saline or sotalol $(10 \mathrm{mg} / \mathrm{kg})(\mathrm{n}=8-10)$, d WT or DBH-CB1KO mice $(\mathrm{n}=5-6)$, e WT or DBH-CB1KO mice after saline or sotalol $(10 \mathrm{mg} / \mathrm{kg})$ treatment $(\mathrm{n}=6-8), \mathbf{f}$ mice pretreated with saline or sotalol $(10 \mathrm{mg} / \mathrm{kg})$ prior to rimonabant (RIM) $(1 \mathrm{mg} / \mathrm{kg})$ or vehicle (VEH) $(\mathrm{n}=9-11)$. Data are expressed as mean \pm s.e.m. Statistical significance was calculated by two-way ANOVA test or Student's t test. 
a

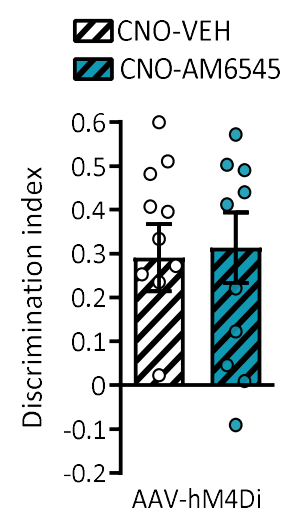

d
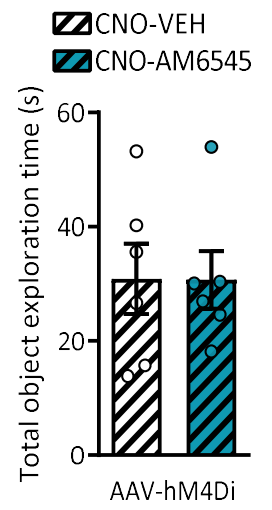

b
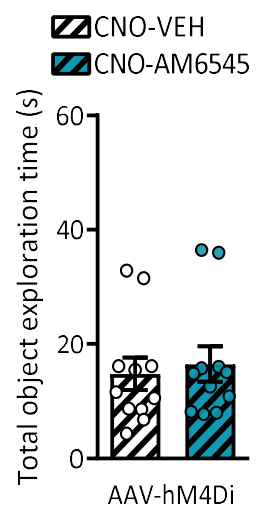

e
C

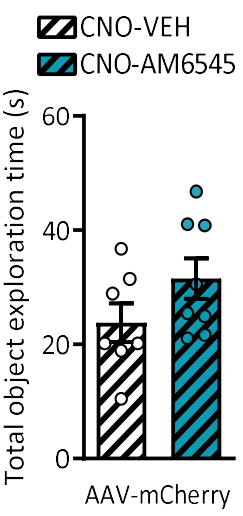

Supplementary Fig. 4. Chemogenetic targeting of the vagus nerve does not modify the behavioral responses in the NORT. a Discrimination index values and $\mathbf{b}$ total exploration times in NORT at $24 \mathrm{~h}$ after the familiarization phase and after CNO (3 $\mathrm{mg} / \mathrm{kg}$ ) and vehicle (VEH) or AM6545 $(1 \mathrm{mg} / \mathrm{kg})$ in mice infected with AAV-hm4Di in the vagus nerve and i.p. $\mathrm{CNO}(3 \mathrm{mg} / \mathrm{kg})$ administration $(\mathrm{n}=10$ - 11) $\mathbf{c}, \mathbf{d}$ Total exploration times in NORT at $48 \mathrm{~h}$ after CNO $(3 \mathrm{mg} / \mathrm{kg}$ ) and vehicle (VEH) or AM6545 $(1 \mathrm{mg} / \mathrm{kg})$ administration in mice infected with c AAV-mCherry $(n=6-8)$ or d AAV-hM4Di ( $=$ 6 - 8). e Representative images of mCherry expression detected in nodose ganglion of mice after AAV5-mCherry injection in the vagus nerve (left image scale bar $=100 \mu \mathrm{m}$, right image scale bar $=30 \mu \mathrm{m})$. Data are expressed as mean \pm s.e.m. Statistical significance was calculated by Student's $t$ test. 


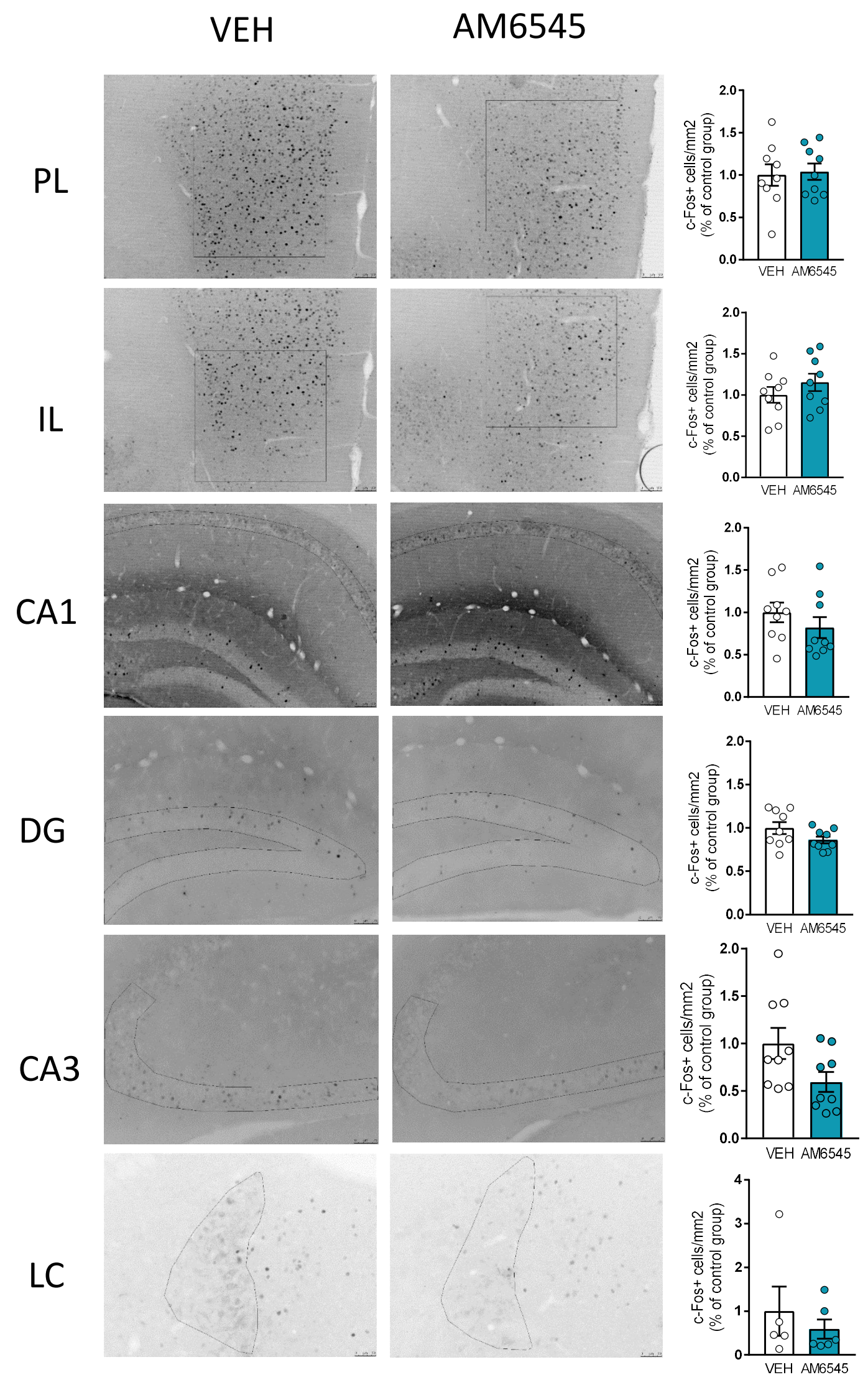




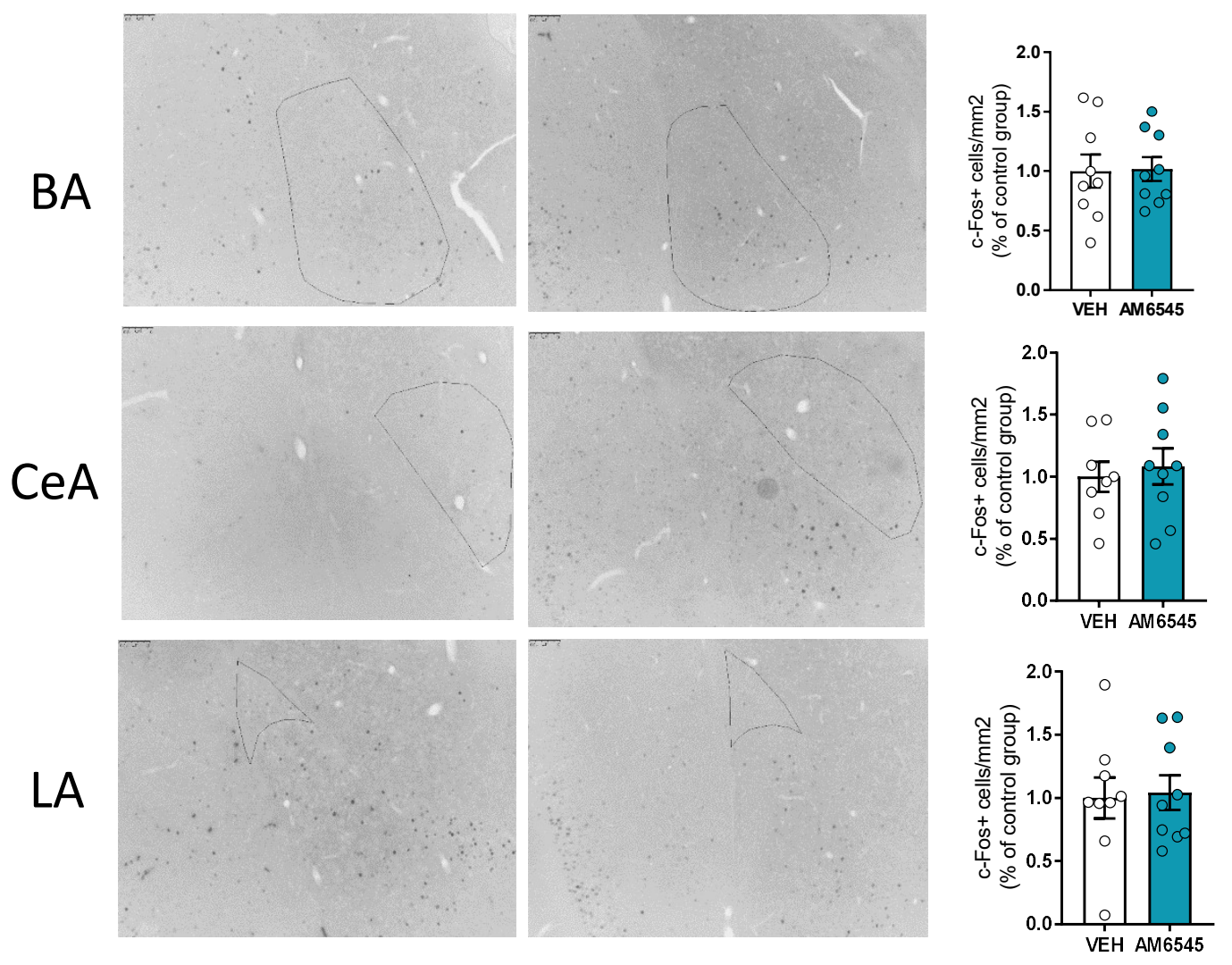

Supplementary Fig. 5. Effects of acute AM6545 treatment in c-Fos expression in specific regions of the CNS. Representative immunohistochemical staining of c-Fos positive cells $90 \mathrm{~min}$ after acute vehicle (VEH) or AM6545 (1 mg/kg) administration in mice $(n=5-9)$. Brain regions evaluated: prelimbic cortex (PL), infralimbic cortex (IL), cornu ammonis 1 (CA1), dentate gyrus (DG), cornu ammonis 3 (CA3), locus coeruleus (LC), basal amygdala (BA), central amygdala (CeA) and lateral amygdala (LA). Data are expressed as mean \pm s.e.m. Statistical significance was calculated by Student's $t$ test. 
a

\section{Vehicle}

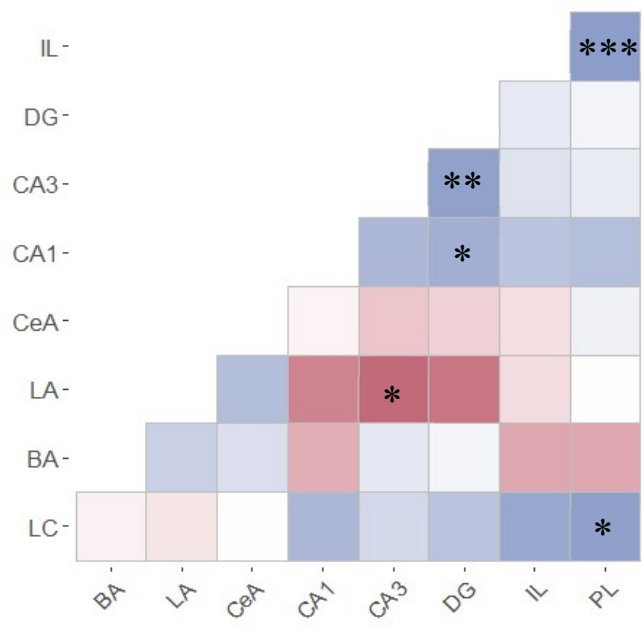

b

AM6545

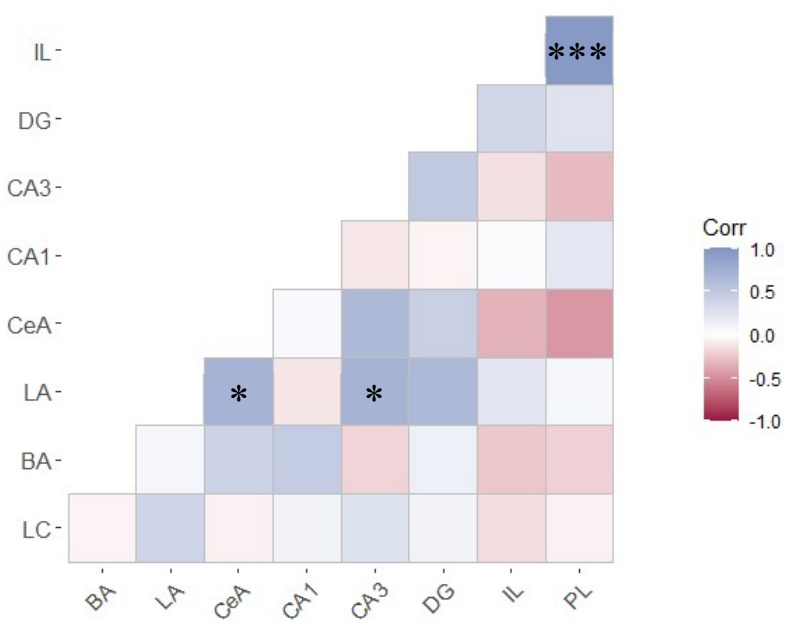

Supplementary Fig. 6. Correlation heat map of c-Fos expression in different brain regions after acute AM6545 administration. Pearson correlation analysis of c-Fos expression in a vehicle and b AM6545 $(1 \mathrm{mg} / \mathrm{kg})$ between different brain areas, including prelimbic cortex (PL), infralimbic cortex (IL), cornu ammonis 1 (CA1), dentate gyrus (DG), cornu ammonis 3 (CA3), locus coeruleus (LC), basal amygdala (BA), central amygdala $(\mathrm{CeA})$ and lateral amygdala (LA). ${ }^{*} \mathrm{p}<0.05,{ }^{*} \mathrm{p}<0.01, * * * \mathrm{p}<0.001$. 


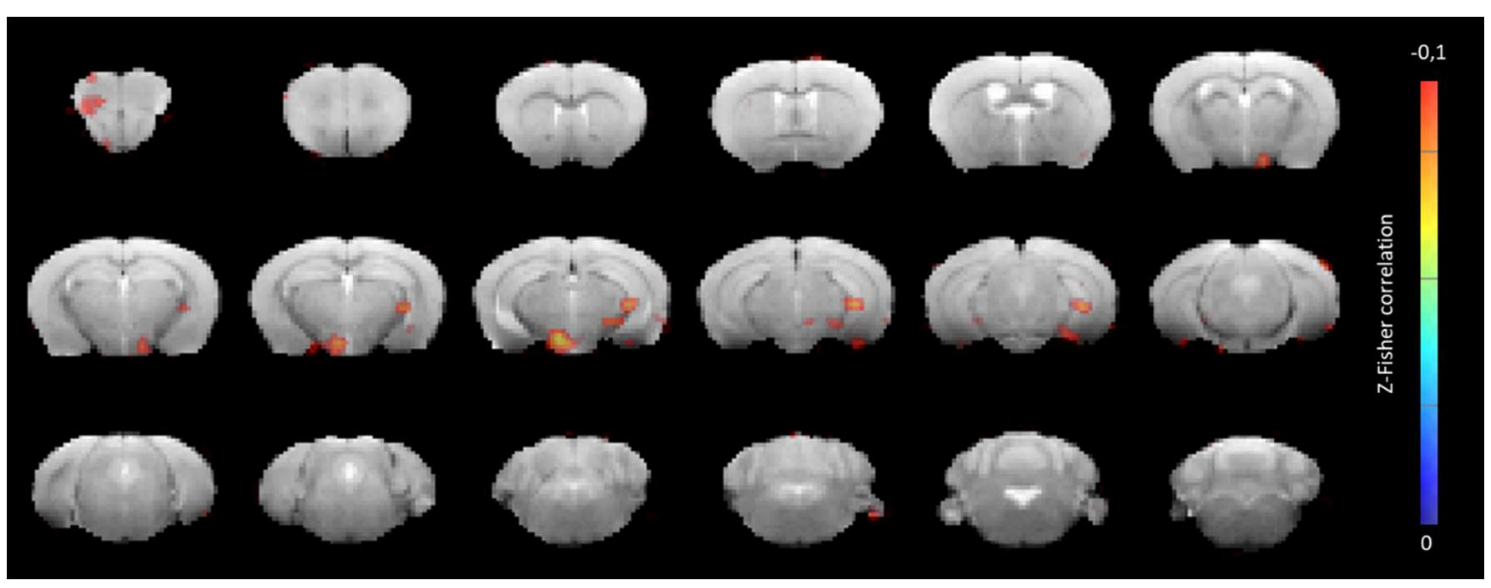

Supplementary Fig. 7. Brainstem connectivity map in AM6545-treated mice: only areas where BOLD signal is negatively correlated to the brainstem signal are shown. Color represents z-Fisher of the correlation coefficient. 
a

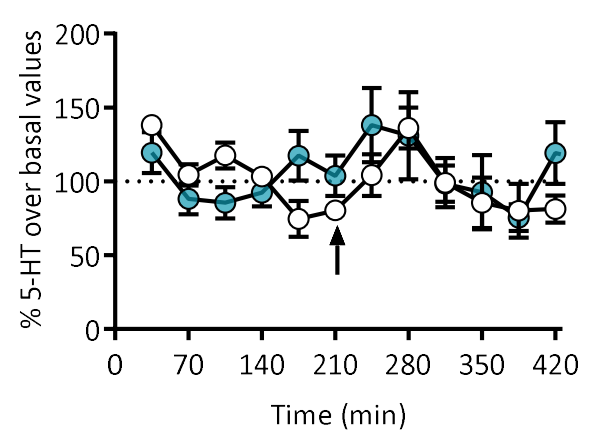

b

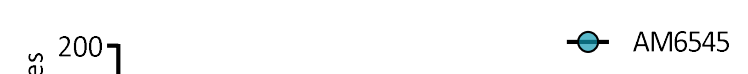

Supplementary Fig. 8. Acute AM6545 administration does not modify other monoamine extracellular levels in the hippocampus. a-b Extracellular a serotonin (5HT) and $\mathbf{b}$ dopamine (DA) levels in the hippocampus after acute AM6545 (1 mg/kg) or vehicle $(\mathrm{VEH})$ administration relative to baseline values $(n=6-7)$. Arrow indicates the time of administration. Points represent mean \pm s.e.m and are expressed as percentages of baseline. Statistical significance was calculated by two-way repeated measures ANOVA test. 
a

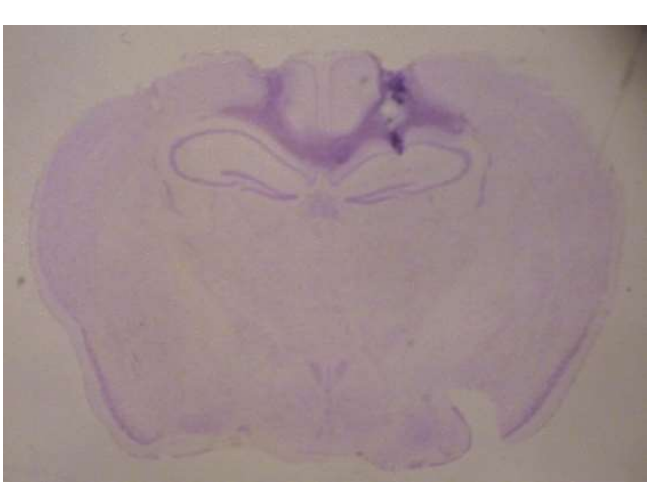

b

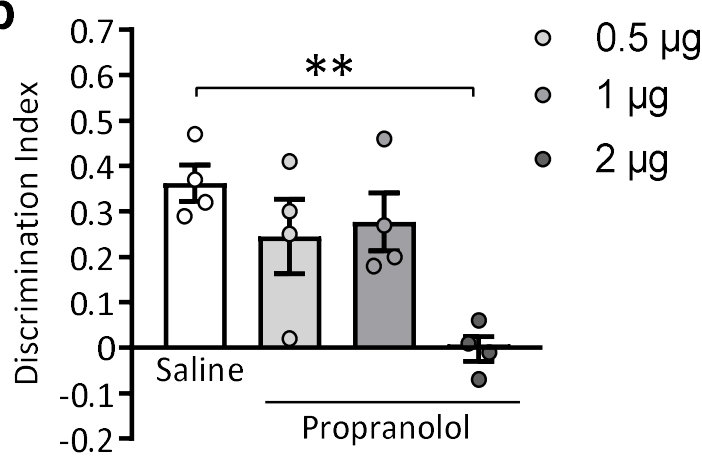

Supplementary Fig. 9. Intrahippocampal propranolol injection. a Cannula placement in the dorsal hippocampus. A brain coronal section from a representative mouse showing cannula placement in the dorsal hippocampus. Brain slices were stained with cresyl violet. b Discrimination index values of different doses of intrahippocampal propranolol infusion or saline in the NORT at $24 \mathrm{~h}(\mathrm{n}=4)$. Data are expressed as mean \pm s.e.m. ${ }^{* *} \mathrm{p}$ $<0.01$ by one-way ANOVA test followed by Tukey post hoc. 